\title{
Nonnegative Matrix Factorization Based
}

\section{Hyperspectral Unmixing with Partially Known}

\section{Endmembers}

\author{
Lei Tong, Jun Zhou, Senior Member, IEEE, Yuntao Qian, Member, IEEE, Xiao \\ Bai, and Yongsheng Gao, Senior Member, IEEE
}

\begin{abstract}
Hyperspectral unmixing is an important technique for estimating fractions of various materials from remote sensing imagery. Most unmixing methods make the assumption that no prior knowledge of endmembers is available before the estimation. This is, however, not true for some unmixing tasks for which part of the endmember signatures may be known in advance. In this paper, we address the hyperspectral unmixing problem with partially known endmembers. We extend nonnegative matrix factorization (NMF) based unmixing algorithms to incorporate prior information into their models. The proposed approach uses the spectral signature of known endmembers as a constraint, among others, in the unmixing model, and propagate the knowledge by an optimization process which minimizes the difference between image data and the prior knowledge. Results on both synthetic and real data have validated the effectiveness of the proposed method, and shown that it has outperformed several state-of-the-art methods that use or do not use prior knowledge of endmembers.
\end{abstract}

\section{Index Terms}

Hyperspectral Unmixing, Nonnegative Matrix Factorization, Partially Known Endmembers.

L. Tong and Y. Gao are with School of Engineering, Griffith University, Nathan, Australia.

J. Zhou is with School of Information and Communication Technology, Griffith University, Nathan, Australia. Correspondence author: J. Zhou (jun.zhou@griffith.edu.au)

Y. Qian is with the Institute of Artificial Intelligence, College of Computer Science, Zhejiang University, Hangzhou 310027, P.R. China.

X. Bai is with the School of Computer Science and Engineering, Beihang University, Beijing 100191, China. 


\section{INTRODUCTION}

Hyperspectral remote sensing images have been widely used for earth monitoring, land cover classification, agriculture, and mining because of the spatial and spectral information contained in the imagery [1], [2], [3], [4]. They can be captured by spectral imaging sensors mounted on satellites, airplanes, and unmanned aerial vehicles. Due to the long distance of the imaging sensors to the targets of monitoring and intrinsic property of sensors, hyperspectral images normally do not have high spatial resolution, which causes the mixed responses of various types of ground objects. Hyperspectral unmixing is an effective method to address this problem. It decomposes mixed pixels into a collection of spectral signatures, i.e., endmembers, and their corresponding proportions, i.e. abundance, at each pixel [5].

Depending on how much prior knowledge on endmembers is known in advance, unmixing methods can be divided into three categories, i.e., no prior knowledge, complete prior knowledge, and partial prior knowledge. In the past few decades, a large number of hyperspectral unmixing methods have been proposed with the assumption that no prior knowledge on endmember is available [6], [7], except the number of endmembers. N-FINDR [8] is one of the earliest unmixing methods, which finds the maximum volume of the simplex to fit the feature data and treats each vertex as an endmember. The pixel purity index (PPI) method [9] is a semi-supervised method that generates the candidates of the endmembers by projecting the hyperspectral data to different unit vectors called skewers. Then, users select the real endmembers from the candidates. Vertex component analysis (VCA) [10] analyses the endmembers using data projection. Simplex identification via split augmented Lagrangian (SISAL) [11] enforces the endmembers to compose a convex hull which contains all pixels in the image data, and adopts a sequence of augmented Lagrangian optimizations.

Nonnegative matrix factorization (NMF) [12] has also attracted much attention. It treats the unmixing as a blind source separation problem, and decomposes image data into two matrices, an endmember matrix and an abundance matrix, simultaneously. However, NMF algorithm may fall into local minima because the objective function of NMF is a non-convex function. One solution is to add constraints to the NMF model. Miao and Qi [13] proposed an NMF method which estimates the endmembers with a minimum volume constraint. The results showed that this method can be used on highly mixed data. Jia and Qian [14] proposed to force the smoothness 
of the endmember and the abundance. Following the same rationale, sparsity of abundance matrix [15] and its manifold regularized extension [16] have both been used to generate sparse abundance solutions. Other constraints on the NMF model include neighborhood information of pixels [17] and dissimilarity of signatures [18].

Some methods assume that sufficient prior knowledge of endmembers is known in advance. This can be in the form of an over-complete spectral library, for example, that provided by the United States Geological Survey [19], or selected endmembers that have been observed in a region. Roberts et. al. [20] selected reference endmembers from a spectral library collected in the field and laboratory. The effectiveness of endmember combination was evaluated based on the unmixing errors of selected two or three endmember mixtures. The linear sparse regression model has also been proposed to estimate the proportion of each endmember in the library [21], [22]. Iordache et. al. [23] introduced a robust sparse representation method which selects few endmembers out of a large library to represent a hyperspectral image. This method also allows the incorporation of spatial contextual information [24]. Akhtar et. al. [25] pointed out that sparse coding is only needed to operate on selected bands at which the variances of material reflectance is high. Fu et al [26] dealt with the problem when the signatures in the library and in the real data are mismatched. Such discrepancy can be incorporated into a multiple signal classification based sparse unmixing method [27] and makes the sparse regression algorithm more robust.

The problem with these approaches is that even though a large endmember dictionary is available as prior information, some key endmembers may still be missed in this dictionary. In real applications, it is often the case that limited prior knowledge, but not all, is available on the area of study. For example, users may know that an area has certain land covers whose spectral signatures can be retrieved from a standard library or by ground survey. Such signatures can even be retrieved directly from image regions that contain only pure endmembers, e.g., in the middle of a road or at the center of a meadow. Therefore, it is necessary to consider the scenarios when only partially known endmembers are available. To explore how such partial prior knowledge can be utilized, Tang and Shi [28] proposed an NMF method which separates the endmember matrix into two parts, one with known endmember signatures extracted from the standard library, and the other with unknown endmembers. An extension of this method utilizes the sparse coding approach on an over-complete dictionary, but assumes that prior knowledge on certain endmembers existing in the image is available [29]. However, these approaches ignore 
the discrepancies between the known endmember signatures and the actual endmember data that can be extracted from the hyperspectral image. Such discrepancies may be caused by weather or ground condition, and by within-class variations of land covers. Directly replacing part of the endmember matrix using known endmembers makes the methods incapable of adapting to the real data being analyzed. To solve this problem, Sigurdsson et. al. [30] pointed out that the estimated endmembers shall be allowed to deviate from known endmembers. A regularization term is used to control such deviation from the prior knowledge.

In this paper, a novel NMF method with partially known endmembers is described. We propose a model which minimizes the differences between the spectral signatures of endmembers being estimated in the image data and the standard signatures of known endmembers extracted from the library. The benefit of this method is that it not only uses the prior knowledge on the unmixing tasks, but also considers the distribution of the real data in the hyperspectral dataset, so that the discrepancy between the prior knowledge and the data can be compromised. Furthermore, the proposed method is general in nature, and can be easily extended to other NMF based hyperspectral unmixing algorithms, such as $L_{1 / 2}$ sparsity [15] and manifold regularization [16].

Our idea is related to the work of Sigurdsson et. al. [30]. However, our method adopts a more flexible setting so that both known and unknown endmembers are included in the modelling formulation. This allows us to develop an integrated optimization method to solve the estimation problem for both types of endmembers. The advantage of this formulation also comes from its extendability to other NMF methods as shown in this paper. On the contrary, applying the method of Sigurdsson et. al. to other NMF models is not straightforward as constraint is only put on the known endmembers. Note that the RMUSIC [26] method also allows flexibility in endmember estimation, which is implemented by finding a vector to compensate the error term. This allows the modelling process to adapt to endmember mismatches between dictionary and the estimation. However, it requires a complete dictionary for the unmixing.

The rest of this paper is organized as follows. In Section II, the linear mixed model and nonnegative matrix factorization method are introduced. Section III describes the proposed NMF method based on partially known endmembers. Section IV extends this method to cope with $L_{1 / 2}$ sparsity constraint and manifold regularized constraint based NMF methods. It also discusses the relationship between our method and two alternative approaches that also use prior knowledge. Section V presents the experimental results on both synthetic and remote sensing data. Finally, 
conclusions are drawn in Section VI.

\section{UNMIXING BASED ON NONNEGATIVE MatRIX FACTORIZATION}

In this section, we describe a linear spectral mixture model (LMM) and its corresponding hyperspectral unmixing solution based on the NMF method. These form the basis for the proposed method on hyperspectral unmixing with partially known prior knowledge.

In a hyperspectral remote sensing image, each pixel can be considered as the mixture of several endmembers. Let the number of wavelength-indexed bands in an image be $H$ and the number of endmembers be $K$. A pixel $\mathbf{y}$ in a hyperspectral image is an $H \times 1$ column vector whose entries correspond to the observed reflectance of objects in different bands. Then $\mathbf{y}$ can be approximated by a linear combination of endmembers

$$
\mathbf{y}=\mathrm{Mr}+\mathbf{e}
$$

where $\mathbf{M}$ is an $H \times K$ matrix $\left(\mathbf{m}_{1}, \ldots, \mathbf{m}_{j}, \ldots, \mathbf{m}_{K}\right)$, in which $\mathbf{m}_{j}$ is an $H \times 1$ column vector representing the spectral signature of the $j^{\text {th }}$ endmember. $\mathbf{r}$ is a $K \times 1$ column vector for endmember abundances, and $\mathbf{e}$ is the additive Gaussian white noise.

It is straightforward to extend the above pixel-level mixing model to the whole image, such that

$$
\mathbf{Y}=\mathbf{M R}+\mathbf{E}
$$

where matrices $\mathbf{Y} \in \mathbb{R}_{+}^{H \times N}, \mathbf{R} \in \mathbb{R}_{+}^{K \times N}$, and $\mathbf{E} \in \mathbb{R}_{+}^{H \times N}$ represent the hyperspectral image, the abundance matrix, and the additive noise, respectively. $N$ is the total number of pixels in image Y.

When hyperspectral unmixing is treated as a blind source separation problem, the only known matrix is $\mathbf{Y}$, while matrices $\mathbf{M}$ and $\mathbf{R}$ are the targets of unmixing methods. According to linear mixture model, two constraints can be naturally imposed on the $\mathbf{M}$ and $\mathbf{R}$ matrices. The first is abundance sum-to-one constraint, which makes the total contribution from each endmember sum to unit. The second is nonnegative constraint, which implies that entries in both endmember signatures and abundances shall be larger than or equal to zero.

Based on the nonnegative constraint, nonnegative matrix factorization (NMF) [31], [32] has been adopted for hyperspectral unmixing. It tries to estimate the endmember matrix $\mathbf{M}$ and abundance matrix $\mathbf{R}$ simultaneously to reconstruct the high dimensional data matrix $\mathbf{Y}$. Based 
on Equation (2), the goal of reconstruction is to minimize the difference between the image data $\mathrm{Y}$ and reconstructed image MR. Therefore, an objective function can be defined as follows:

$$
\operatorname{obj}(\mathbf{M}, \mathbf{R})=\frac{1}{2}\|\mathbf{Y}-\mathbf{M R}\|_{F}^{2}
$$

where $\|\cdot\|_{F}$ represents the Frobenius norm.

A common method for minimizing this objective function is multiplied iterative algorithm [32], [12]. When applied to Equation (3), the multiplicative rule is given by the following two equations:

$$
\begin{aligned}
& \mathbf{M} \leftarrow \mathbf{M} \cdot * \mathbf{Y R}^{T} \cdot / \mathbf{M R R}^{T} \\
& \mathbf{R} \leftarrow \mathbf{R} \cdot * \mathbf{M}^{T} \mathbf{Y} \cdot / \mathbf{M}^{T} \mathbf{M R}
\end{aligned}
$$

where $(.)^{T}$ is the transpose of the matrix, .* and.$/$ denote element-wise multiplication and division, respectively.

\section{APPROACH}

In this section, we describe the proposed NMF method based on partially known endmembers and its corresponding optimization algorithm. The proposed method is derived on the basis of NMF and can be extended to other NMF based hyperspectral unmixing methods.

\section{A. NMF with Partially Known Endmembers}

As mentioned earlier, most NMF based unmixing methods have neglected prior knowledge on endmember signatures. However, with the availability of libraries on spectral signatures of various endmembers and other prior knowledge on the areas to be analyzed, the spectral information of some endmembers can be known in advance before the unmixing step. In order to effectively use such prior known endmembers, we propose a new constraint which minimizes the differences between the estimated endmembers and the known endmembers.

Let $\mathbf{M} \in \mathbb{R}_{+}^{H \times K}$ and $\mathbf{B} \in \mathbb{R}_{+}^{H \times K}$ represent the endmember matrix to be estimated and the matrix with partially known endmember signature respectively. Let matrix $\mathbf{B}$ be constructed as follows:

$$
\mathbf{B}=\left[\mathbf{B}_{Q n}, 0, \cdots, 0\right]
$$


where

$$
\mathbf{B}_{Q n}=\left[\mathbf{b}_{1}, \cdots, \mathbf{b}_{q}\right]
$$

are $q$ known endmembers.

One main goal of our method is to minimize the differences between known endmembers and the estimated endmembers using the observed data. This can be done by imposing a new constraint on matrices $\mathbf{M}$ and $\mathbf{B}$. Therefore, the objective function can be defined as follows:

$$
\operatorname{obj}(\mathbf{M}, \mathbf{R})=\frac{1}{2}\|\mathbf{Y}-\mathbf{M R}\|_{F}^{2}+\frac{1}{2} \lambda\|\mathbf{B}-\mathbf{M S}\|_{F}^{2}
$$

where matrix $\mathbf{B}$ contains the partial prior knowledge of endmember spectral, $\lambda \in \mathbb{R}$ is a scalar which weights the contribution of the partial prior knowledge, $\mathbf{S}$ is a matrix with the size of $K \times K$ defined as follows:

$$
\mathbf{S}=\left(\begin{array}{ll}
\mathbf{I} & 0 \\
0 & 0
\end{array}\right)
$$

where matrix $\mathbf{I}$ is an identity matrix of size $q \times q$.

The first term in Equation (8) is the same as the reconstruction error in the NMF method. In the second term, the constraint to the optimization problem is from parts of $\mathbf{M}$ and $\mathbf{B}$ that correspond to known endmembers. If in some cases users have strong confidence that the known endmembers are consistent with some target endmembers in the hyperspectral image, $\lambda$ can be set to a large value to increase the contribution of the prior knowledge.

\section{B. Multiplicative Iterative Algorithm}

In section II, we have described the multiplicative iterative algorithm for the optimization process of NMF approach. In our method, this algorithm is adopted and modified to meet the requirements of the proposed objective function. When the constraint on prior knowledge is incorporated into the objective function, by taking the partial derivative with respect to $\mathbf{M}$, we get:

$$
\frac{\partial \operatorname{obj}(\mathbf{M}, \mathbf{R})}{\partial \mathbf{M}}=-(\mathbf{Y}-\mathbf{M R}) * \mathbf{R}^{T}-\lambda(\mathbf{B}-\mathbf{M} \mathbf{S}) * \mathbf{S}^{T}
$$

Using gradient descent method, $\mathbf{M}$ can be updated as follows:

$$
\mathbf{M} \leftarrow \mathbf{M}-\eta\left(-\mathbf{Y} \mathbf{R}^{T}+\mathbf{M R R ^ { T }}-\lambda(\mathbf{B}-\mathbf{M S}) * \mathbf{S}^{T}\right)
$$


Let

$$
\eta=\frac{\mathbf{M}}{\mathbf{M}\left(\mathbf{R R}^{T}+\lambda \mathbf{S S}^{T}\right)}
$$

the above step becomes:

$$
\left.\mathbf{M} \leftarrow \mathbf{M} . *\left(\mathbf{Y R}^{T}+\lambda \mathbf{B S}^{T}\right) \cdot /\left(\mathbf{M R R}^{T}+\lambda \mathbf{M S S}^{T}\right)\right)
$$

The iteration of $\mathbf{R}$ remains the same as the NMF solution, i.e., it is identical to Equation (5).

\section{Convergence of the Algorithm}

Now, we prove the convergence of the objective function in Equation (8) by our updating algorithm. Since the updating rule for $\mathbf{R}$ is the same as NMF, we only focus on updating Equation (13). To do so, we make use of an auxiliary function similar to that used in the Expectation-Maximization (EM) algorithm [33], [34]. We define an auxiliary function $G\left(\mathbf{m}, \mathbf{m}^{t}\right)$ satisfying the conditions $G(\mathbf{m}, \mathbf{m})=o b j(\mathbf{m})$ and $G\left(\mathbf{m}, \mathbf{m}^{t}\right) \geq o b j(\mathbf{m})$ such that $o b j(\mathbf{m})$ is nonincreasing when updated using the following:

$$
\mathbf{m}^{(t+1)}=\arg \min _{\mathbf{m}} G\left(\mathbf{m}, \mathbf{m}^{t}\right)
$$

This is guaranteed by

$$
\operatorname{obj}\left(\mathbf{m}^{(t+1)}\right) \leq G\left(\mathbf{m}^{(t+1)}, \mathbf{m}^{t}\right) \leq G\left(\mathbf{m}^{t}, \mathbf{m}^{t}\right)=\operatorname{obj}\left(\mathbf{m}^{t}\right)
$$

We define the function $G$ as

$$
G\left(\mathbf{m}, \mathbf{m}^{t}\right)=\operatorname{obj}\left(\mathbf{m}^{t}\right)+\left(\mathbf{m}-\mathbf{m}^{t}\right)\left(\nabla o b j\left(\mathbf{m}^{t}\right)\right)^{T}+\frac{1}{2}\left(\mathbf{m}-\mathbf{m}^{t}\right) \mathbf{K}\left(\mathbf{m}^{t}\right)\left(\mathbf{m}-\mathbf{m}^{t}\right)^{T}
$$

where the diagonal matrix $\mathbf{K}\left(\mathbf{m}^{t}\right)$ is given by

$$
\begin{aligned}
\mathbf{K}\left(\mathbf{m}^{t}\right) & =\operatorname{diag}\left(\mathbf{R}^{T} \mathbf{R} \mathbf{m}^{t}+\lambda \mathbf{S}^{T} \mathbf{S} \mathbf{m}^{t}\right) \cdot / \mathbf{m}^{t} \\
& =\operatorname{diag}\left(\mathbf{R}^{T} \mathbf{R} \mathbf{m}^{t}\right) \cdot / \mathbf{m}^{t}+\operatorname{diag}\left(\lambda \mathbf{S}^{T} \mathbf{S} \mathbf{m}^{t}\right) \cdot / \mathbf{m}^{t}
\end{aligned}
$$

Take derivative of $\mathbf{K}\left(\mathbf{m}^{t}\right)$

$$
\mathbf{K}\left(\mathbf{m}^{t}\right)^{\prime}=\operatorname{diag}\left(\mathbf{R}^{T} \mathbf{R} \mathbf{m}^{t}\right) \cdot / \mathbf{m}^{t}-\mathbf{R}^{T} \mathbf{R}+\operatorname{diag}\left(\lambda \mathbf{S}^{T} \mathbf{S m}^{t}\right) \cdot / \mathbf{m}^{t}-\lambda \mathbf{S}^{T} \mathbf{S}
$$

Lee and Seung [31] have proven the positive semidefiniteness of $\mathbf{K}\left(\mathbf{m}^{t}\right)^{\prime}$, which makes the objective function decrease monotonically at each iteration. Because of the nonnegative property 
of the terms in the objective function, it is a bounded function, i.e., with lower bound 0 , therefore, the objective function converges. However, in terms of convergence of variables, it has been pointed out that the multiplicative iterative algorithm may fail to converge to a stationary point [35], [36]. The convergence of the variables of the algorithm has not been established and should be further investigated in future work.

\section{Implementation Issues}

As mentioned in Section II, the abundance matrix should meet the sum to one constraint. In the implementation, the method in [37] is adopted to apply this constraint. The data matrix $\mathbf{Y}$ and the signature matrix $\mathbf{M}$ are replaced by $\mathbf{Y}_{f}$ and $\mathbf{M}_{f}$ which are augmented matrices by a row of constants defined as follows:

$$
\begin{gathered}
\mathbf{Y}_{f}=\left(\begin{array}{c}
\mathbf{Y} \\
\delta \mathbf{1}_{N}^{T}
\end{array}\right) \\
\mathbf{M}_{f}=\left(\begin{array}{c}
\mathbf{M} \\
\delta \mathbf{1}_{K}^{T}
\end{array}\right)
\end{gathered}
$$

where $\delta$ is a parameter to balance the impact of the additivity constraint on the abundance matrix. A large $\delta$ forces the sum of abundances at each pixel close to unity. In our experiments, we set $\delta$ to 10 . In the iteration, these augmented matrices replace $\mathbf{Y}$ and $\mathbf{M}$ in the update rule of $\mathbf{R}$ given in Equation (5).

For the NMF methods, the initialization of endmember matrix $\mathbf{M}$ and abundance matrix $\mathbf{R}$ affects the result. In our implementation, the initial endmember matrix is computed by the VCA method [10]. The initialization of abundance matrix $\mathbf{R}$ is computed by the inverting of $\mathbf{M}$ as follows

$$
\mathbf{R}=\mathbf{M}^{\#} * \mathbf{Y}
$$

where $\mathbf{M}^{\#}$ is the inverse of $\mathbf{M}$. We force the matrix $\mathbf{R}$ to be non-negative, as follows:

$$
\mathbf{R}=\max (\mathbf{R}, 0)
$$

When assigning the known endmembers to the initial endmember matrix, a greedy algorithm is used [38]. Firstly, the pairwise distances between initial endmembers and known endmembers are calculated. The initial endmember with a smallest distance is replaced with the corresponding 
known endmember. The matching is then performed from the rest of the initial and known endmembers, until every known endmember is assigned a location in the endmember matrix.

There are two stopping criteria in our implementation. The first one is the maximum iteration number which is set to 3000 . The second one is error tolerance defined as follow

$$
\left|\operatorname{obj}\left(\mathbf{M}^{i}, \mathbf{R}^{i}\right)\right| \leq \epsilon\left|\operatorname{obj}\left(\mathbf{M}^{i-1}, \mathbf{R}^{i-1}\right)\right|
$$

where $\epsilon$ is set to $10^{-4}$ in the experiments. Once either of these criteria is met, the optimization process terminates.

The procedure of our partially known endmember unmixing method is described as following.

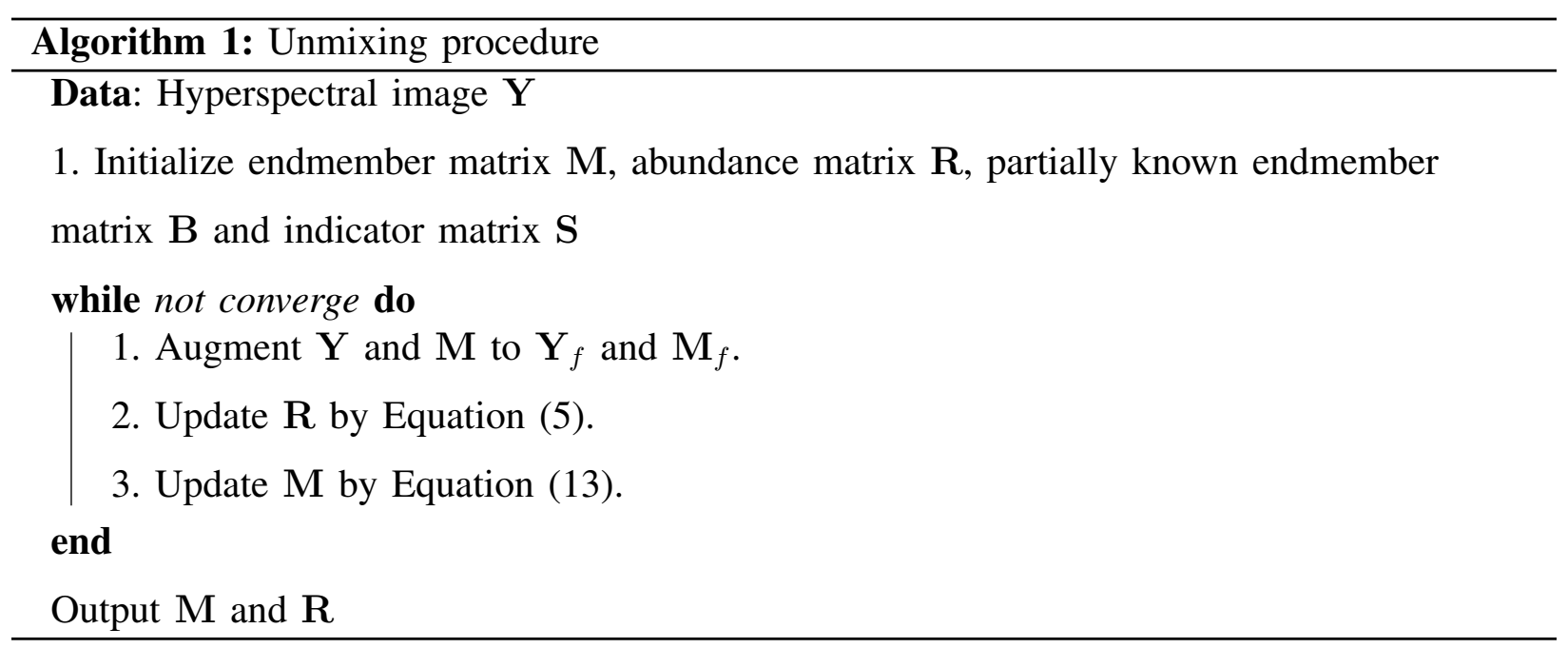

\section{Method Extension And Discussions}

The proposed method makes use of the prior information on known endmembers. It is general in nature and can be extended to other NMF based unmixing methods. In this section, we show two such examples by extending our method to $L_{1 / 2}$ sparsity regularized NMF [15] and manifold regularized sparse NMF [16]. Then we provide a discussion on the differences between our method and an alternative method for NMF based unmixing with Prior Knowledge (UPKNMF) [28] of endmembers and a sparse regression based method which also uses a priori information (SUNSPI) [29]. 
A. $L_{1 / 2}$ sparsity constrained NMF with partially known endmembers

As pointed out by Qian et. al. [15], it is difficult to obtain a globally optimal solution of Equation (3) because of the non-convexity of the objective function. Moreover, NMF lacks a unique solution because $\mathbf{M R}=(\mathbf{M C})\left(\mathbf{C}^{-1} \mathbf{R}\right)$ for any nonnegative invertible matrix $\mathbf{C}$. So sparsity constraint has been introduced to partially solve this problem [39]. Among them, $L_{1 / 2^{-}}$ NMF [15] has demonstrated excellent performance. The objective function of $L_{1 / 2}$-NMF is

$$
\operatorname{obj}(\mathbf{M}, \mathbf{R})=\frac{1}{2}\|\mathbf{Y}-\mathbf{M R}\|_{F}^{2}+\gamma\|\mathbf{R}\|_{\frac{1}{2}}
$$

where $\gamma$ weights the contribution of $\|\mathbf{R}\|_{1 / 2}$, and $\|\mathbf{R}\|_{1 / 2}$ is defined as

$$
\|\mathbf{R}\|_{1 / 2}=\sum_{k=1}^{K} \sum_{n=1}^{N} \mathbf{R}_{k n}^{1 / 2}
$$

When applied to Equation (24), the multiplicative update rule is given by Equation (4) and

$$
\mathbf{R} \leftarrow \mathbf{R} \cdot * \mathbf{M}^{T} \mathbf{Y} \cdot /\left(\mathbf{M}^{T} \mathbf{M R}+\frac{\gamma}{2} \mathbf{R}^{-\frac{1}{2}}\right)
$$

To incorporate the proposed method into $L_{1 / 2}-\mathrm{NMF}$, we add a partially known endmembers constraint to the objective function such that

$$
\operatorname{obj}(\mathbf{M}, \mathbf{R})=\frac{1}{2}\|\mathbf{Y}-\mathbf{M R}\|_{F}^{2}+\frac{1}{2} \lambda\|\mathbf{B}-\mathbf{M S}\|_{F}^{2}+\gamma\|\mathbf{R}\|_{1 / 2}
$$

where $\mathbf{B}$ and $\mathbf{S}$ are defined in Equation (6) and Equation (9) respectively.

The multiplicative iterative algorithm is then updated by

$$
\left.\mathbf{M} \leftarrow \mathbf{M} . *\left(\mathbf{Y R}^{T}+\lambda \mathbf{B}^{T}\right) \cdot /\left(\mathbf{M R R}^{T}+\lambda \mathbf{M S S}^{T}\right)\right)
$$

and Equation (26) for $\mathbf{R}$. The convergence of this objective function can be proven in a similar manner as in the $L_{1 / 2}$ sparsity regularized NMF paper [15].

\section{B. Manifold regularized Sparse NMF with partially known endmembers}

Sparsity constrained NMF only considers the sparse characteristics in the abundance matrix during the modelling. Some other intrinsic properties of abundances can also be adopted, for example, the relationship between the abundances of different pixels in the image. This is the rationale of manifold regular constraint [16] which enables the pixels with similar spectral 
signatures to have similar abundance. The objective function of the manifold regularized sparse NMF is

$$
\operatorname{obj}(\mathbf{M}, \mathbf{R})=\frac{1}{2}\|\mathbf{Y}-\mathbf{M R}\|_{F}^{2}+\gamma\|\mathbf{R}\|_{1 / 2}+\frac{\mu}{2} \operatorname{Tr}\left(\mathbf{R} \mathbf{L} \mathbf{R}^{T}\right)
$$

where $\mu \in \mathbb{R}_{+}$is a scalar which weights the contribution of manifold constraint. $\operatorname{Tr}()$ represents the trace of a matrix. To compute the manifold constraint matrix $\mathbf{L}$, a weight matrix $\mathbf{W}$ and a diagonal matrix $\mathbf{D}$ should be computed in advance. Let $\mathbf{y}_{i}$ be one of the k-nearest neighbors of $\mathbf{y}_{j}$, where $\mathbf{y}_{i}$ and $\mathbf{y}_{j}$ are two samples, the weight $\mathbf{W}_{i j}$ is assigned using a heat kernel

$$
\mathbf{W}_{i j}=\exp -\frac{\left\|\mathbf{y}_{i}-\mathbf{y}_{j}\right\|^{2}}{\sigma}
$$

It is apparent that if the $\mathbf{y}_{i}$ and $\mathbf{y}_{j}$ are similar, the value of $\mathbf{W}_{i j}$ is relatively large.

The diagonal matrix $\mathbf{D}$ and manifold constraint matrix $\mathbf{L}$ are computed as follows:

$$
\begin{aligned}
\mathbf{D}_{i i} & =\sum_{j} \mathbf{W}_{i j} \\
\mathbf{L} & =\mathbf{D}-\mathbf{W}
\end{aligned}
$$

In order to incorporate the prior knowledge into the objective function, we add a partially known endmembers constraint, such that the object function with manifold regularized sparse NMF becomes:

$$
o b j(\mathbf{M}, \mathbf{R})=\frac{1}{2}\|\mathbf{Y}-\mathbf{M R}\|_{F}^{2}+\frac{1}{2} \lambda\|\mathbf{B}-\mathbf{M S}\|_{F}^{2}+\gamma\|\mathbf{R}\|_{1 / 2}+\frac{\mu}{2} \operatorname{Tr}\left(\mathbf{R} \mathbf{L} \mathbf{R}^{T}\right)
$$

where $\lambda, \gamma$ and $\mu \in \mathbb{R}_{+}$are scalar weights. $\mathbf{M}$ and $\mathbf{B}$ are the estimated endmember matrix and known endmember matrix respectively. The multiplicative iterative algorithm is then updated by Equation (28) and

$$
\mathbf{R} \leftarrow \mathbf{R} \cdot *\left(\mathbf{M}^{T} \mathbf{Y}+\mu \mathbf{R W}\right) \cdot /\left(\mathbf{M}^{T} \mathbf{M R}+\frac{\gamma}{2} \mathbf{R}^{-\frac{1}{2}}+\mu \mathbf{R D}\right)
$$

for the endmember and abundance estimation respectively.

\section{Discussions}

Several other unmixing models also use prior knowledge in the estimation process. We briefly discuss two methods here. The first method is UPK-NMF which is also an NMF based unmixing 
method which copes with prior knowledge of spectral signatures [28]. In UPK-NMF, the objective function is defined as follows:

$$
o b j=\frac{1}{2}\left\|\mathbf{Y}-\mathbf{M}_{1} \mathbf{R}_{1}-\mathbf{M}_{2} \mathbf{R}_{2}\right\|^{2}
$$

where $\mathbf{M}_{1}$ and $\mathbf{M}_{2}$ are the matrices of known and unknown endmembers in the size of $H \times Q$ and $H \times(K-Q)$, respectively. $\mathbf{R}_{1}$ and $\mathbf{R}_{2}$ are the matrices of known and unknown abundance matrices in the size of $Q \times N$ and $(K-Q) \times N$, respectively. The optimization solution includes the following update rules

$$
\begin{aligned}
& \mathbf{M}_{2} \leftarrow \mathbf{M}_{2} *\left(\mathbf{Y} \mathbf{R}_{2}^{T}\right) \cdot /\left(\mathbf{M}_{2} \mathbf{R}_{2} \mathbf{R}_{2}^{T}+\mathbf{M}_{1} \mathbf{R}_{1} \mathbf{R}_{2}^{T}\right) \\
& \mathbf{R}_{1} \leftarrow \mathbf{R}_{1} \cdot *\left(\mathbf{M}_{1}^{T} \mathbf{Y}\right) \cdot /\left(\mathbf{M}_{1}^{T} \mathbf{M}_{1} \mathbf{R}_{1}+\mathbf{M}_{1}^{T} \mathbf{M}_{2} \mathbf{R}_{2}\right) \\
& \mathbf{R}_{2} \leftarrow \mathbf{R}_{2} *\left(\mathbf{M}_{2}^{T} \mathbf{Y}\right) \cdot /\left(\mathbf{M}_{2}^{T} \mathbf{M}_{2} \mathbf{R}_{2}+\mathbf{M}_{2}^{T} \mathbf{M}_{1} \mathbf{R}_{1}\right)
\end{aligned}
$$

Both UPK-NMF and our method make use of the prior knowledge of endmembers. However, there are three main differences between them. First, UPK-NMF simply replaces the estimated endmembers with known endmembers. Our method, on the other hand, sets a constraint to minimize the differences between the estimated endmember and the known one. This leads to the benefit that when there are discrepancies between the standard signature and the corresponding one estimated from the data, our method shows more flexibility in compromising the prior knowledge and the data. Second, UPK-NMF method only updates the unknown parts of the endmember, while in our method, both known and unknown parts are updated. When there are discrepancies between the ground truth and prior knowledge, our method can update the known part while the UPK-NMF method cannot. Last, our method is a general method which can be easily extended to other NMF based unmixing algorithms. Only one constraint needs to be added to the abundance matrices of those variations of NMF. UPK-NMF, however, updates the known and unknown parts of the abundance matrix separately. This makes the extension to other NMF based models difficult as constraints on the abundance matrix are normally applied to the whole matrix. In this paper, we have shown two examples in combining the new constraint with the $L_{1 / 2}$-NMF method and the MRS-NMF method. UPK-NMF, on the contrary, does not have such flexibility.

The second method to be discussed here is the SUNSPI [29] method which is a state-of-the-art hyperspectral unmixing method that uses prior known endmembers based on a sparse regression 
method. An over-complete dictionary of signatures is adopted with a part of the signatures replaced by the known endmembers. The objective function of SUNSPI is defined as follows:

$$
\min _{\mathbf{R}} \frac{1}{2}\|\mathbf{A R}-\mathbf{Y}\|_{F}^{2}+\lambda_{S} \sum_{i=1}^{K}\left\|\mathbf{r}_{i}\right\|+\lambda_{P} \sum_{i \in A / P}\left\|\mathbf{r}^{i}\right\|_{2}
$$

where $\mathbf{A}$ is the dictionary containing signatures and $\mathbf{R}$ is the abundance matrix. $\lambda_{S} \geq 0$ and $\lambda_{P} \geq 0$ are the weights for regularization terms. $\mathbf{r}_{i}$ and $\mathbf{r}^{i}$ are the $i$ th column and $i$ th row of $\mathbf{R}$ respectively. In this method, the over-complete signature library $\mathbf{A}$ is divided to two sets, a known signature set $(P)$ and an unknown signature set $(A / P)$. The abundance of set $A / P$, the unknown set, should be row-sparse while set $P$ should not be row-sparse. This method is fundamentally different from our method. First, SUNSPI is based on sparse regression, but our method is based on NMF. Second, SUNSPI requires an over-complete spectral library. Our method only requires known endmembers and does not set requirements on the unknown ones. Third, SUNSPI focuses on estimating the abundance matrix only but our method estimates both endmembers and their abundances.

\section{EXPERIMENTS}

Having presented our method in the previous sections, we now demonstrate its effectiveness in unmixing. A series of experiments have been done to evaluate the performance of the proposed methods based on the basic NMF method (NMF-PPK) and extended versions with $L_{1 / 2}$ sparsity constraint $\left(L_{1 / 2}-\mathrm{PPK}\right)$ and manifold regularized sparse constraint (MRS-PPK). We compared our methods with several alternative methods on both synthetic and real hyperspectral data. These included the basic NMF method (NMF), $L_{1 / 2}$ sparsity regularized NMF $\left(L_{1 / 2}-\mathrm{NMF}\right)[15]$, NMF with manifold regularized sparse constraint (MRS-NMF) [16], NMF unmixing with prior knowledge (UPK-NMF) [28], sparse coding approaches CLSUNSAL [23], and SUNSPI [29]. The codes of UPK-NMF, CLSUNSAL, and SUNSPI were downloaded from their author's websites, and the other algorithms were implemented by us using Matlab.

In the experiments, two metrics are used to evaluate the performance of different methods: the spectral angle distance (SAD) and the root mean squared error (RMSE). The spectral angle distance is used to compare the similarity between the ground truth end-member $\mathbf{M}_{k}$ and the 


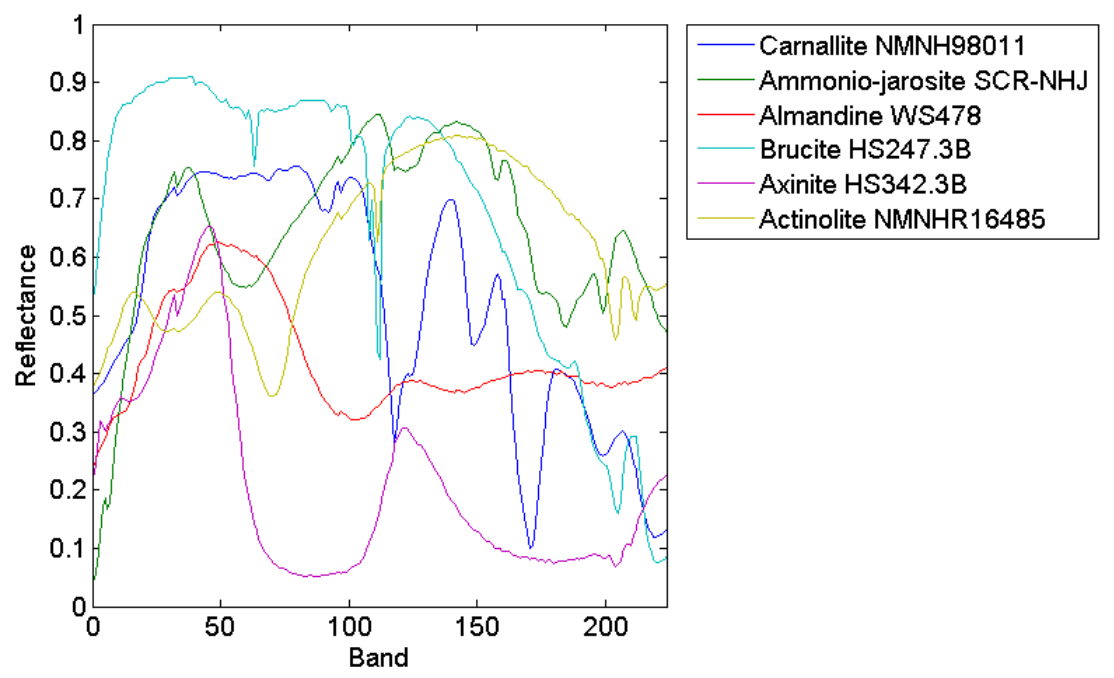

Fig. 1. Example endmember spectral used in synthetic data.

estimated endmember $\widehat{\mathbf{M}}_{k}$, which is defined as

$$
\mathrm{SAD}_{k}=\arccos \left(\frac{\mathbf{M}_{k}^{T} \widehat{\mathbf{M}}_{k}}{\left\|\mathbf{M}_{k}\right\|\left\|\widehat{\mathbf{M}}_{k}\right\|}\right)
$$

The root mean square error (RMSE) is used to evaluate the accuracy of the estimated abundance. It is defined as

$$
\mathrm{RMSE}_{k}=\left(\frac{1}{N}\left|\mathbf{R}_{k}-\widehat{\mathbf{R}}_{k}\right|^{2}\right)^{\frac{1}{2}}
$$

where $\widehat{\mathbf{R}}_{k}$ is the ground-truth abundance matrix for the $k^{\text {th }}$ endmember.

\section{A. Experiments on Synthetic Data}

We first show the results on synthetic data on which we know the ground truth of both endmember signatures and their abundances at each pixel. To generate the synthetic data, six spectral signatures are chosen from the USGS digital spectral library [19]. These spectral signatures are from Carnallite, Ammonio-jarosite, Almandine, Brucite, Axinite and Actinolite, which are shown in Fig. 1. The synthetic image was generated in a similar way as in [13]. The image contains $64 \times 64$ pixels, which is divided into $8 \times 8$ regions. In each region the same type of ground cover was initialized with one of the endmembers chosen randomly. Then a low pass filter of $9 \times 9$ was applied to generate mixed data and a threshold $\theta=0.7$ was used to control the extent 


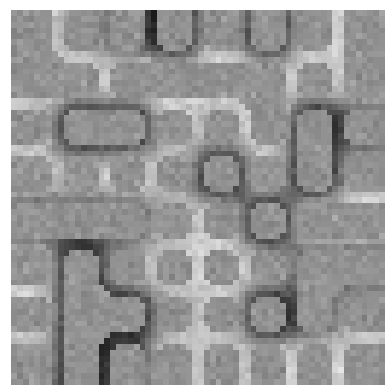

(a)

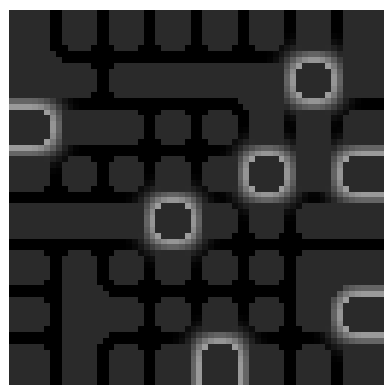

(b)

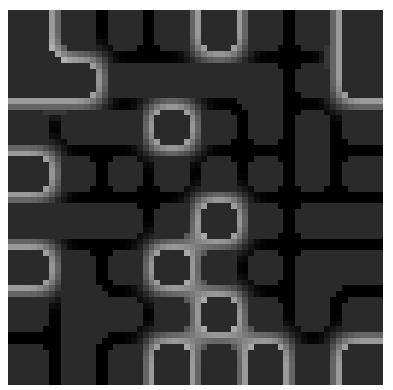

(c)

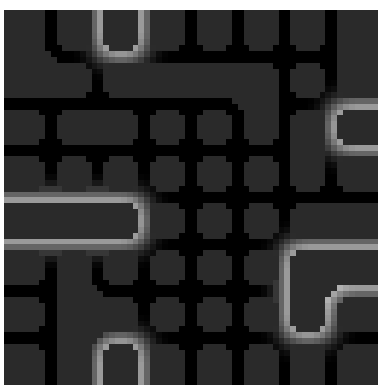

(d)

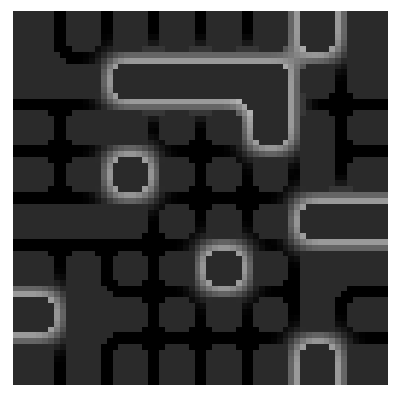

(e)

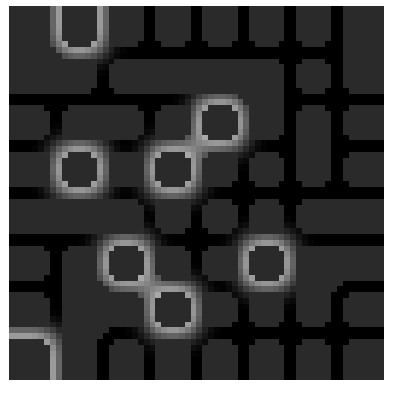

(f)

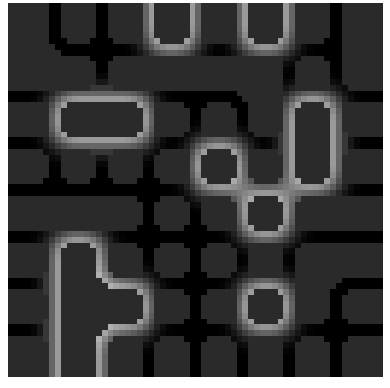

(g)

Fig. 2. Synthetic data. (a) Synthetic data of band 100; (b)-(g) ground truth abundance maps for six endmembers.

of mixing. For a pixel at which the abundance of an arbitrary endmember is larger than $\theta$, the abundance of endmembers at this pixel is replaced with a mixture of all endmembers with equal abundances. Finally, to evaluate the robustness to noise, zero-mean Gaussian noise is added to synthetic data. The signal to noise ratio (SNR) is defined as

$$
S N R=10 \log _{10} \frac{E\left[y^{T} y\right]}{E\left[e^{T} e\right]}
$$

where $\mathbf{y}$ and $\mathbf{e}$ are the observation and noise of a pixel. $E[\cdot]$ denotes the expectation operator. The default setting of SNR is 25 on the synthetic data. Fig. 2(a) displays the synthetic data of band 100 with SNR of $25 \mathrm{~dB}$. Fig. 2(b)-(g) show the abundance maps of six endmembers.

1) Experiment 1 (Contribution of Prior Knowledge): The first experiment evaluates the impact of $\lambda$ which controls the contribution of the prior knowledge in unmixing. In this experiment, NMF-PPK is used to perform the unmixing, with known endmembers randomly selected. The SNR is set to $25 \mathrm{~dB}$. We ran our experiments on the synthetic data with the initial endmember and abundance matrices generated following the method described in section III-D. In our method, all endmembers are estimated, even though prior knowledge on some endmembers is given. Fig. 3(a) 


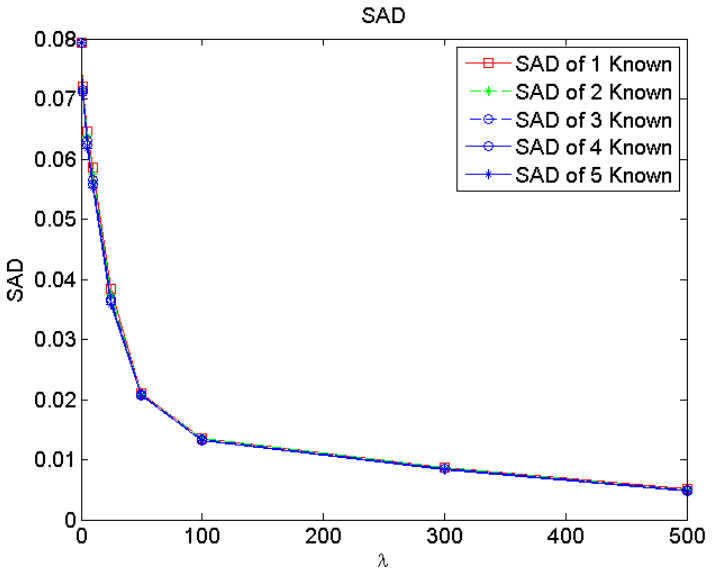

(a)

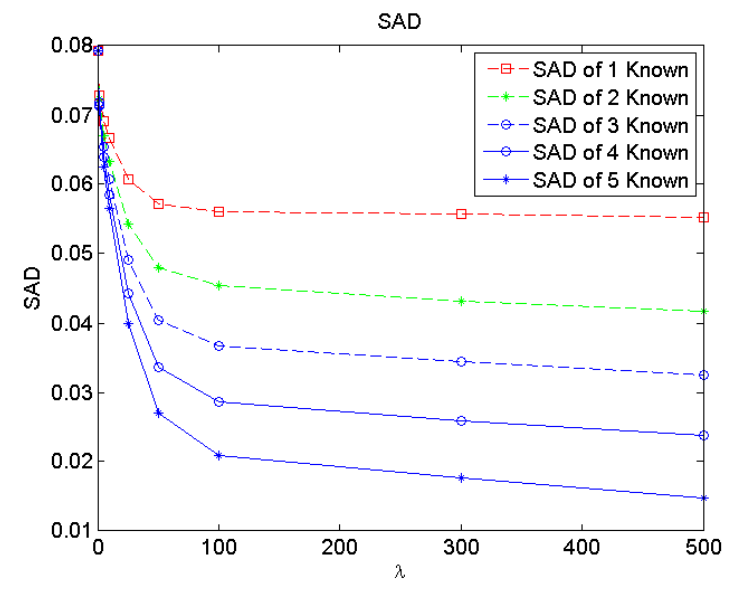

(c)

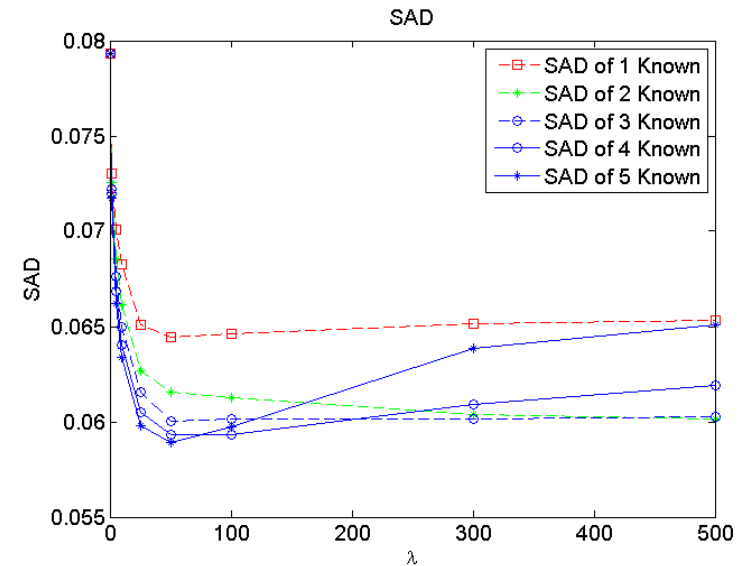

(b)

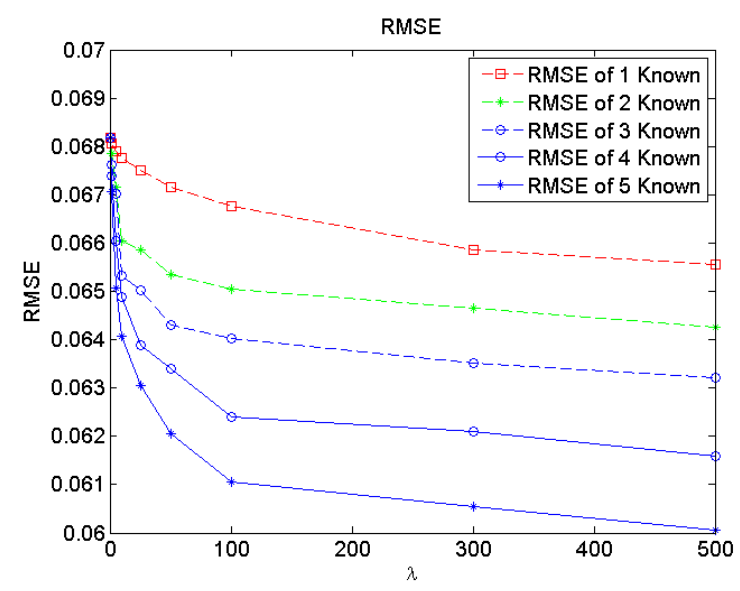

(d)

Fig. 3. Impact of $\lambda$ on the SADs between the estimated endmembers and ground truth of (a) known endmembers, (b) unknown endmembers, and (c) all endmembers. (d) shows the RMSE of the estimated abundance matrix of all endmembers.

and 3(b) show the mean SADs between the estimated signatures and the known endmembers, and between the estimated signatures and ground truth of unknown endmembers respectively, when different values of $\lambda$ are set. Fig. 3(c) shows the overall mean estimation errors of both known and unknown endmembers. Fig. 3(d) shows the root mean square errors of estimated abundance of all endmembers.

It is natural to see that the larger $\lambda$ is, the higher similarity is forced between the estimated and the known endmembers. Therefore, the SAD decreases with the increase of $\lambda$ value in Fig. 3(a). 
However, due to the noise and discrepancy between ground truth known endmembers and the estimated signatures from the data, increasing $\lambda$ does not always improve the accurate estimation of unknown endmembers. It can be seen in Fig. 3(b) when $\lambda$ increases, that the SAD decreases first then increases in most cases. On the one hand, this suggests that introducing appropriate prior knowledge can increase the accuracy of the endmember estimation. On the other hand, this also implies that the model should not rely too much on the known endmembers when there are discrepancies between the prior knowledge and the data. This is particularly the case when a large number of endmembers are known, for which the SAD increases quickly when $\lambda$ is larger than 50. Therefore, the contribution of the prior knowledge term and the data term in the unmixing model shall be compromised. Based on this experiment, setting $\lambda$ from 20 to 100 is appropriate. We set $\lambda$ to 50 in the following experiments, unless otherwise specified. When the overall SAD of both known and unknown endmember estimations are considered, as displayed in Fig. 3(c), a continuously decreasing SAD can also be observed. The overall values, however, fall between the estimates of known and unknown endmembers. A similar trend can be observed on the RMSE results displayed in Fig. 3(d).

2) Experiment 2 (Comparison of Methods With and Without Prior Knowledge): The purpose of this experiment is to compare the proposed method with several baseline methods. These include NMF, $L_{1 / 2}-\mathrm{NMF}$, and MRS-NMF which do not use prior knowledge, and UPK-NMF which is an alternative method that uses prior knowledge. We also compare our method with VCA which is used to initialize the proposed method. To demonstrate the advantage of using prior knowledge, we report the mean SADs of all estimated endmembers and RMSEs of all estimated abundances. To make a fair comparison with those methods without using prior knowledge, we also report the mean SADs of the estimated unknown endmembers only. For those methods that use sparsity constraint, the control parameter $\gamma$ is computed in the same way as in Qian et. al. [15]. For the MRS method, we set $\mu=0.1$ which is a suggested value in its original paper [16]. For other parameters in each method being compared, we manually tuned them to optimal. We varied the number of endmembers that were used as prior knowledge. In practice, some endmembers tend to generate higher errors than others. To reduce the influence of random known endmember selection in evaluating different methods, we enumerated all known endmember combinations for each setting. For each combination, we ran the experiments ten times with different initial endmember and abundance values generated by VCA. Then, we calculated the mean values and 
TABLE I

MEAN SADS AGAINST THE NUMBER OF KNOWN ENDMEMBERS ON SYNTHETIC DATA.

\begin{tabular}{l|c|c|c|c|c}
\hline \hline $\begin{array}{l}\text { Number of Known End- } \\
\text { members }\end{array}$ & 1 & 2 & 3 & 4 & 5 \\
\hline \hline VCA & $0.176 \pm 0.012$ & $0.176 \pm 0.012$ & $0.176 \pm 0.012$ & $0.176 \pm 0.012$ & $0.176 \pm 0.012$ \\
\hline NMF & $0.079 \pm 0.012$ & $0.079 \pm 0.012$ & $0.079 \pm 0.012$ & $0.079 \pm 0.012$ & $0.079 \pm 0.012$ \\
NMF-PPK (Unknown) & $0.073 \pm 0.009$ & $0.068 \pm 0.008$ & $0.065 \pm 0.008$ & $0.062 \pm 0.009$ & $0.061 \pm 0.010$ \\
NMF-PPK (All) & $0.067 \pm 0.009$ & $0.057 \pm 0.008$ & $0.049 \pm 0.007$ & $0.043 \pm 0.007$ & $0.037 \pm 0.008$ \\
\hline $\begin{array}{l}L_{1 / 2}-\mathrm{NMF} \\
L_{1 / 2}-\mathrm{PPK} \text { (Unknown) }\end{array}$ & $0.077 \pm 0.007$ & $0.073 \pm 0.008$ & $0.070 \pm 0.009$ & $0.068 \pm 0.010$ & $0.067 \pm 0.011$ \\
$L_{1 / 2}$-PPK (All) & $0.071 \pm 0.006$ & $0.062 \pm 0.006$ & $0.054 \pm 0.006$ & $0.047 \pm 0.006$ & $0.041 \pm 0.005$ \\
\hline MRS & $0.081 \pm 0.009$ & $0.081 \pm 0.009$ & $0.081 \pm 0.009$ & $0.081 \pm 0.009$ & $0.081 \pm 0.009$ \\
MRS-PPK (Unknown) & $0.076 \pm 0.005$ & $0.073 \pm 0.005$ & $0.070 \pm 0.006$ & $0.067 \pm 0.008$ & $0.066 \pm 0.009$ \\
\hline $\begin{array}{l}\text { MRS-PPK (All) } \\
\text { UPK-NMF (Unknown) }\end{array}$ & $0.077 \pm 0.005$ & $0.073 \pm 0.005$ & $0.072 \pm 0.009$ & $0.076 \pm 0.018$ & $0.090 \pm 0.020$ \\
\hline \hline
\end{tabular}

standard deviations of SAD and RMSE.

Tables I and II show the mean SADs and mean RMSEs of each method. It can be seen that our methods clearly outperformed all corresponding baseline methods that do not use prior knowledge, i.e., NMF-PPK vs NMF, $L_{1 / 2}$-NMF vs $L_{1 / 2}$-PPK, and MRS-PPK vs MRS-NMF. This proves that prior knowledge is useful for unmixing. The advantages of our method are significant when all estimated endmembers are used for SAD evaluation. When the number of known endmembers increases, the mean values of SAD on all endmembers and RMSE decrease. This is consistent with the results reported in Fig. 3(c) and (d). In the case of SAD on unknown endmembers, the SAD values are also improved compared to the case where no prior knowledge is used. This experiment suggests that effective use of prior knowledge can improve unmixing performance.

Our method also outperforms UPK-NMF when unknown endmembers are evaluated. NMFPPK shows marginal improvement over UPK-NMF on abundance estimation as well. This implies 
TABLE II

MEAN RMSES AGAINST THE NUMBER OF KNOWN ENDMEMBERS ON SYNTHETIC DATA.

\begin{tabular}{l|c|c|c|c|c}
\hline \hline $\begin{array}{l}\text { Number of Known End- } \\
\text { members }\end{array}$ & 1 & 2 & 3 & 4 & 5 \\
\hline \hline $\mathrm{VCA}$ & $0.106 \pm 0.012$ & $0.106 \pm 0.012$ & $0.106 \pm 0.012$ & $0.106 \pm 0.012$ & $0.106 \pm 0.012$ \\
\hline $\mathrm{NMF}$ & $0.068 \pm 0.009$ & $0.068 \pm 0.009$ & $0.068 \pm 0.009$ & $0.068 \pm 0.009$ & $0.068 \pm 0.009$ \\
$\mathrm{NMF-PPK}$ & $0.067 \pm 0.009$ & $0.065 \pm 0.009$ & $0.064 \pm 0.009$ & $0.063 \pm 0.009$ & $0.062 \pm 0.009$ \\
\hline$L_{1 / 2}-\mathrm{NMF}$ & $0.078 \pm 0.012$ & $0.078 \pm 0.012$ & $0.078 \pm 0.012$ & $0.078 \pm 0.012$ & $0.078 \pm 0.012$ \\
$L_{1 / 2}-\mathrm{PPK}$ & $0.076 \pm 0.012$ & $0.075 \pm 0.012$ & $0.074 \pm 0.012$ & $0.073 \pm 0.012$ & $0.072 \pm 0.012$ \\
\hline $\mathrm{MRS}$ & $0.076 \pm 0.013$ & $0.076 \pm 0.013$ & $0.076 \pm 0.013$ & $0.076 \pm 0.013$ & $0.076 \pm 0.013$ \\
$\mathrm{MRS}-\mathrm{PPK}$ & $0.075 \pm 0.012$ & $0.074 \pm 0.012$ & $0.073 \pm 0.012$ & $0.072 \pm 0.012$ & $0.071 \pm 0.012$ \\
\hline $\mathrm{UPK}-\mathrm{NMF}$ & $0.072 \pm 0.011$ & $0.069 \pm 0.011$ & $0.067 \pm 0.011$ & $0.065 \pm 0.011$ & $0.067 \pm 0.011$ \\
\hline \hline
\end{tabular}

that proper usage of the prior knowledge is important. UPK-NMF has neglected the discrepancy between prior knowledge and real data. When noises is present in the image, and when there is within-class variance of endmembers, UPK-NMF cannot adapt to such a condition. This result also proves that it is necessary to use the parameter $\lambda$ in Equation (8) to adjust the contribution of prior knowledge. Nonetheless, the UPK-NMF method has achieved the best results when SAD is calculated from all endmembers. This is natural because the errors on the known endmembers are zero in UPK-NMF. It should also be mentioned here that, due to the low sparsity of the synthetic data and initialization method, the performance of the NMF based method is better than those counterparts with sparsity constraints.

3) Experiment 3 (Robustness to Noise): In this experiment, we test the robustness of our methods against different signal to noise ratio (SNR) from $15 \mathrm{~dB}$ to $45 \mathrm{~dB}$ with $10 \mathrm{~dB}$ in steps. We also set infinity SNR to simulate the noise free condition. In the experiment, four out of the six endmembers were set as the prior knowledge. We also enumerated all combinations of four known endmembers. In this case, it is 15 combinations. Similarly, we reported the mean and standard deviation values of the different methods. The mean SADs and mean RMSEs are shown in Tables III and IV respectively.

It can be seen from the results that the NMF-PPK, $L_{1 / 2}$-PPK, MRS-PPK methods have 
TABLE III

MEAN SADS AGAINST DIFFERENT SNRS ON SYNTHETIC DATA.

\begin{tabular}{l|c|c|c|c|c}
\hline \hline SNR & 15 & 25 & 35 & 45 & $\infty$ \\
\hline \hline VCA & $0.189 \pm 0.025$ & $0.176 \pm 0.012$ & $0.150 \pm 0.012$ & $0.168 \pm 0.013$ & $0.167 \pm 0.021$ \\
\hline NMF & $0.137 \pm 0.036$ & $0.079 \pm 0.012$ & $0.073 \pm 0.007$ & $0.074 \pm 0.015$ & $0.067 \pm 0.006$ \\
NMF-PPK (Unknown) & $0.105 \pm 0.008$ & $0.062 \pm 0.009$ & $0.070 \pm 0.008$ & $0.050 \pm 0.017$ & $0.047 \pm 0.005$ \\
NMF-PPK (All) & $0.055 \pm 0.003$ & $0.043 \pm 0.007$ & $0.047 \pm 0.008$ & $0.032 \pm 0.014$ & $0.021 \pm 0.002$ \\
\hline$L_{1 / 2}$-NMF & $0.135 \pm 0.034$ & $0.082 \pm 0.005$ & $0.074 \pm 0.005$ & $0.073 \pm 0.023$ & $0.080 \pm 0.010$ \\
$L_{1 / 2}$-PPK (Unknown) & $0.102 \pm 0.007$ & $0.068 \pm 0.010$ & $0.069 \pm 0.008$ & $0.063 \pm 0.012$ & $0.057 \pm 0.007$ \\
$L_{1 / 2}$-PPK (All) & $0.057 \pm 0.002$ & $0.047 \pm 0.006$ & $0.049 \pm 0.009$ & $0.040 \pm 0.009$ & $0.029 \pm 0.003$ \\
\hline MRS & $0.134 \pm 0.034$ & $0.081 \pm 0.009$ & $0.074 \pm 0.005$ & $0.073 \pm 0.023$ & $0.080 \pm 0.009$ \\
MRS-PPK (Unknown) & $0.101 \pm 0.008$ & $0.067 \pm 0.008$ & $0.069 \pm 0.008$ & $0.063 \pm 0.011$ & $0.057 \pm 0.008$ \\
MRS-PPK (All) & $0.057 \pm 0.003$ & $0.046 \pm 0.003$ & $0.049 \pm 0.009$ & $0.041 \pm 0.010$ & $0.029 \pm 0.004$ \\
\hline UPK-NMF (Unknown) & $0.119 \pm 0.015$ & $0.076 \pm 0.018$ & $0.094 \pm 0.018$ & $0.086 \pm 0.031$ & $0.063 \pm 0.024$ \\
UPK-NMF (All) & $0.039 \pm 0.005$ & $0.025 \pm 0.006$ & $0.032 \pm 0.006$ & $0.029 \pm 0.009$ & $0.020 \pm 0.008$ \\
\hline \hline
\end{tabular}

outperformed their baseline methods NMF, $L_{1 / 2}-\mathrm{NMF}$, and MRS-NMF respectively. Significant improvement on endmember estimation can be achieved, no matter if all endmembers or unknown endmembers are evaluated. Improvement on abundance estimation can also be observed, though such improvement is marginal. This experiment implies that unmixing with prior knowledge on endmembers leads to clear advantages. When the SNR value becomes smaller, especially changing from $25 \mathrm{~dB}$ to $15 \mathrm{~dB}$, the $\mathrm{SAD}$ and RMSE values become larger for all the methods. This means that noise is an important factor to consider in unmixing tasks, as a low noise level generally leads to improved unmixing accuracy. UPK-NMF has achieved the best performance on SAD of all endmembers. However, when evaluated on SAD of unknown endmembers and RMSE of abundance estimation, our methods have significantly outperformed UPK-NMF. This shows how important it is for the estimation method to adapt to the discrepancy between prior knowledge and real data.

4) Experiment 4 (Discrepancy between Known Endmembers and Ground Truth Endmembers): In this experiment, we simulate the circumstances in which there is discrepancy between known 
TABLE IV

MEAN RMSES AGAINST DIFFERENT SNRS ON SYNTHETIC DATA.

\begin{tabular}{l|c|c|c|c|c}
\hline \hline $\mathrm{SNR}$ & 15 & 25 & 35 & 45 & $\infty$ \\
\hline \hline $\mathrm{VCA}$ & $0.132 \pm 0.020$ & $0.106 \pm 0.012$ & $0.115 \pm 0.007$ & $0.123 \pm 0.012$ & $0.120 \pm 0.014$ \\
\hline $\mathrm{NMF}$ & $0.106 \pm 0.010$ & $0.068 \pm 0.009$ & $0.068 \pm 0.017$ & $0.063 \pm 0.023$ & $0.064 \pm 0.010$ \\
$\mathrm{NMF-PPK}$ & $0.098 \pm 0.008$ & $0.063 \pm 0.009$ & $0.065 \pm 0.018$ & $0.053 \pm 0.034$ & $0.060 \pm 0.008$ \\
\hline$L_{1 / 2}-\mathrm{NMF}$ & $0.109 \pm 0.012$ & $0.078 \pm 0.012$ & $0.079 \pm 0.019$ & $0.074 \pm 0.024$ & $0.070 \pm 0.011$ \\
$L_{1 / 2}-\mathrm{PPK}$ & $0.100 \pm 0.009$ & $0.073 \pm 0.012$ & $0.075 \pm 0.020$ & $0.064 \pm 0.023$ & $0.064 \pm 0.010$ \\
\hline $\mathrm{MRS}$ & $0.108 \pm 0.012$ & $0.076 \pm 0.013$ & $0.079 \pm 0.019$ & $0.074 \pm 0.024$ & $0.071 \pm 0.011$ \\
$\mathrm{MRS}-\mathrm{PPK}$ & $0.099 \pm 0.008$ & $0.072 \pm 0.012$ & $0.076 \pm 0.020$ & $0.064 \pm 0.023$ & $0.064 \pm 0.010$ \\
\hline $\mathrm{UPK}-\mathrm{NMF}$ & $0.102 \pm 0.010$ & $0.065 \pm 0.011$ & $0.066 \pm 0.016$ & $0.089 \pm 0.040$ & $0.063 \pm 0.014$ \\
\hline \hline
\end{tabular}

TABLE V

COMPARISON OF UPK-NMF AND NMF-PPK WHEN THERE IS DISCREPANCY BETWEEN KNOWN ENDMEMBERS AND GROUND TRUTH ENDMEMBERS.

\begin{tabular}{ccc}
\hline \hline & UPK-NMF & NMF-PPK \\
\hline SAD & $0.140 \pm 0.049$ & $0.088 \pm 0.010$ \\
RMSE & $0.168 \pm 0.023$ & $0.092 \pm 0.014$ \\
\hline \hline
\end{tabular}

endmembers and ground truth endmembers, and compare the performance of UPK-NMF and NMF-PPK. In the dataset, the AluniteGDS84 shown in Fig. 4 was used to generate the synthetic date with other endmembers. However, AluniteGDS83 shown in Fig. 4 was treated as the endmember known prior. We set the total number of endmembers to 4 , and $S N R=25 d B$.

The experimental results are summarized in Table V. We can see that our method is better than UPK-NMF. This is because UPK-NMF simply uses the wrong endmember to unmix the data, causing worse performance. Whereas, in our method, the negative influence can be alleviated by combining the contributions from the data term and the prior knowledge term in the objective function. 


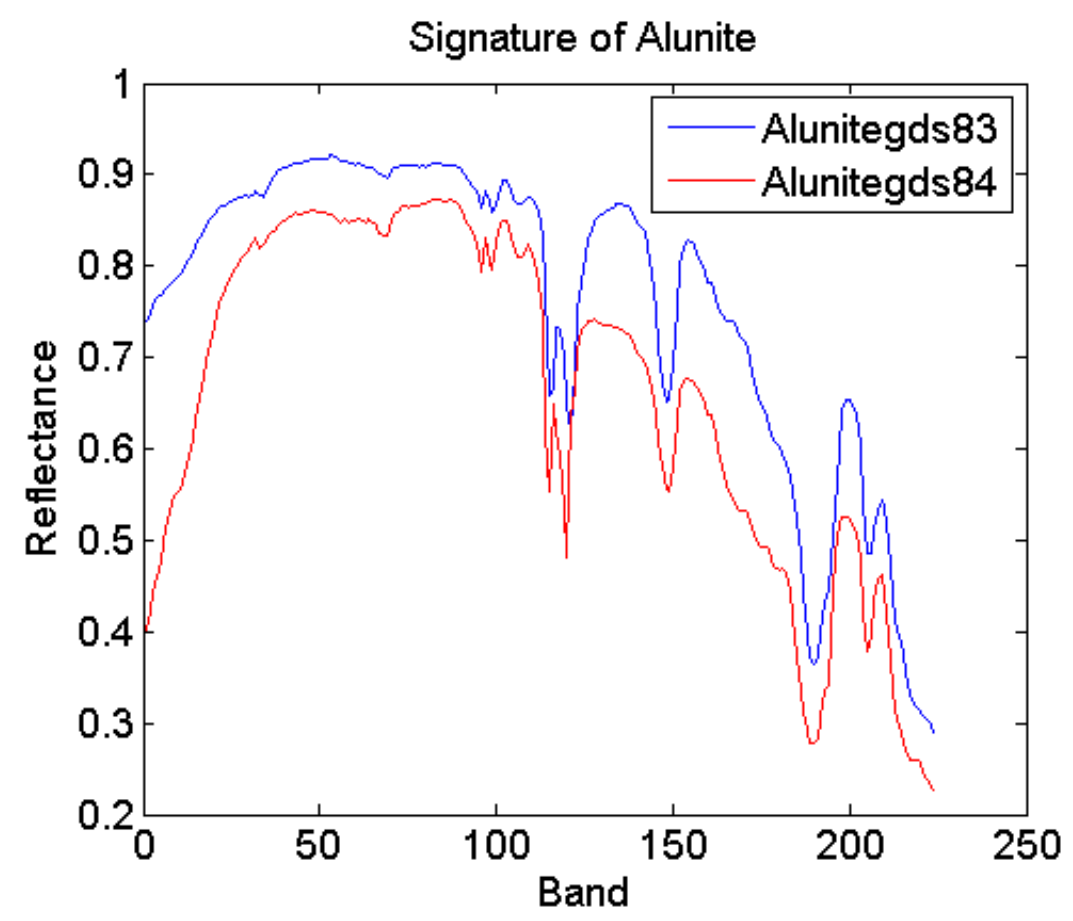

(a)

Fig. 4. Spectral signatures of endmembers used for discrepancy analysis. AluniteGDS83 is used to construct the synthetic data and AluniteGDS84 is treated as the prior knowledge.

5) Experiment 5 (Compare with Sparse Regression Method): The purpose of this experiment is to compare our method with the sparse coding methods. Two state-of-the-art methods are selected here: CLSUNSAL [23] and SUNSPI [29]. CLSUNSAL [23] reconstructs the hyperspectral image using an over-complete spectral dictionary that can be seen as the full prior knowledge on endmembers. The unmixing process automatically selects endmembers from the dictionary and estimates their abundance. SUNSPI [29] is a sparse coding method that incorporates prior knowledge into the over-complete dictionary in the unmixing model as introduced in Section IV.

In this experiment, we used 6 endmembers, which are the same as in the previous experiments, to generate the synthetic data. For the CLSUNSAL and SUNSPI methods, we used 498 signatures from the USGS digital spectral library [19] to form an over-complete dictionary. For classes not included in the USGS library, such as tree and road, we used the manually collected ground truth from the image. We set 5 endmembers as the prior knowledge for NMF-PPK and SUNSPI, and compare their RMSE values with those from CLSUNSAL in different SNRs from $15 \mathrm{~dB}$ to $25 \mathrm{~dB}$. 


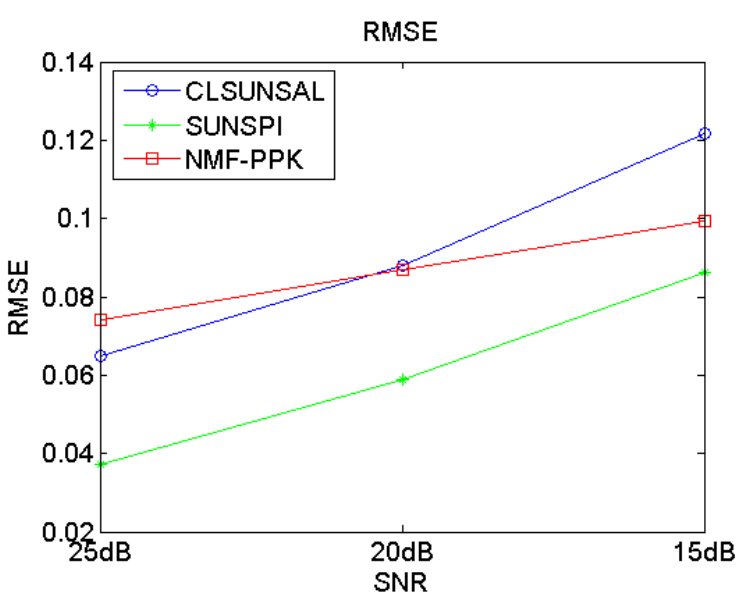

(a)

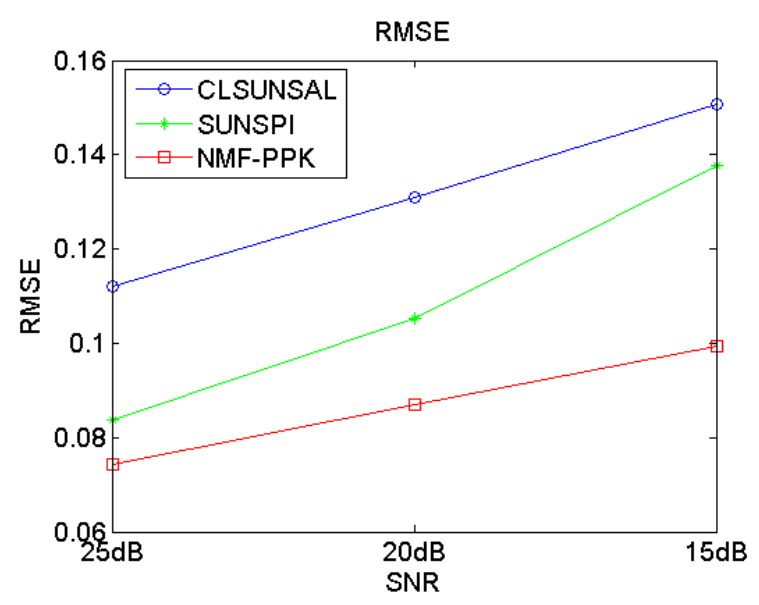

(b)

Fig. 5. (a) RMSE of CLSUNSAL, SUNSPI and $L_{1 / 2}$-PPK. (b) RMSE of CLSUNSAL, SUNSPI with incomplete dictionary and $L_{1 / 2}$-PPK.

The RMSEs of CLSUNSAL and SUNSPI are calculated between the estimated abundances of 6 endmembers used to generate the synthetic data and their ground truth abundances. We tuned $\lambda$ from 0.1 to 1 with an interval of 0.1 and chose the best result for the CLSUNSAL method. We used the same tuning procedure on $\lambda_{p}$ and $\lambda_{S}$ for the SUNSPI method. The tolerance for the CLSUNSAL and SUNSPI methods were set to 1e-4 and $\mu=0.1$. Fig. 5(a) shows that the performance of the SUNSPI method is consistently better than CLSUNSAL and NMF-PPK. The CLSUNSAL method is better than NMF-PPK when SNR is high.

The problem with the CLSUNSAL and SUNSPI methods is that they assume all endmembers are available in the dictionary. However, in reality, it is often difficult to cover all materials with one spectral library. In this case, an incomplete dictionary may affect the accuracy of abundance estimation. We conducted an experiment to simulate such a condition by removing two signatures of Carnallite ${ }^{1}$ from the dictionary, and used only 496 signatures in CLSUNSAL and SUNSPI. Fig. 5 shows that the performance of the CLSUNSAL and SUNSPI methods are significantly deteriorated with an incomplete dictionary, which leads to much higher RMSEs than NMF-PPK which relies on partial prior knowledge.

\footnotetext{
${ }^{1}$ Note that Carnallite NMNH98011 was used to construct the synthetic data.
} 


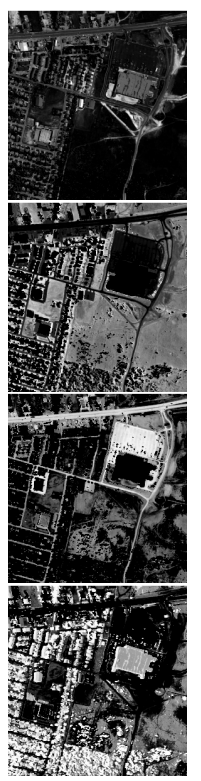

(a) $L_{1 / 2}-\mathrm{NMF}$

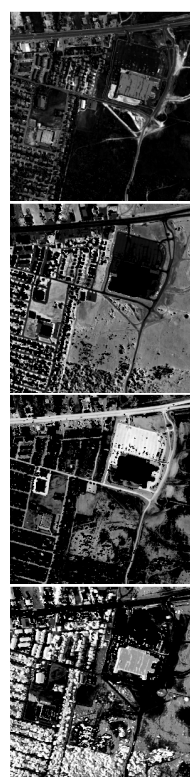

(b) $L_{1 / 2}$-PPK

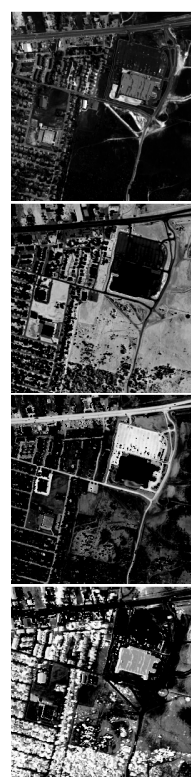

(c) MRS

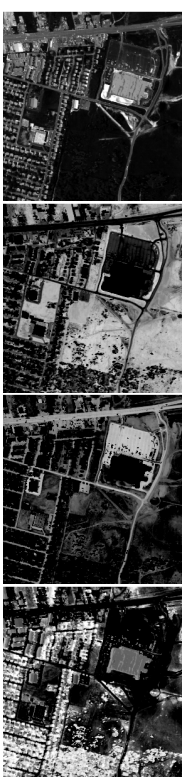

(d) MRS-PPK
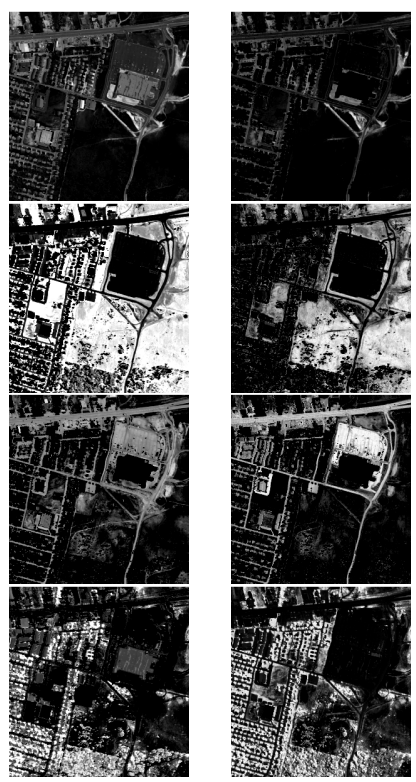

(e) UPK-NMF

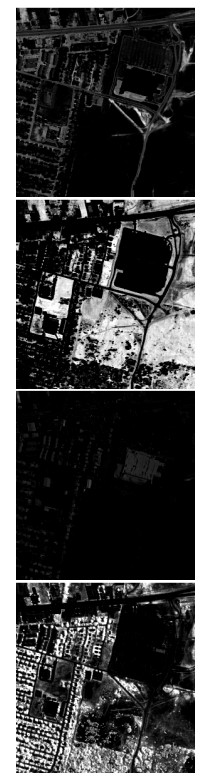

(g) SUNSPI

Fig. 6. Results of abundance estimation on the Urban HYDICE dataset. From top to bottom, rows show the abundance of Road, Grass, Asphalt and Tree, respectively.

\section{B. Experiments on Remote Sensing Data}

To validate the performance of the proposed methods, we also ran experiments on three realworld hyperspectral datasets, i.e., Urban HYDICE, Jasper Ridge, and Cuprite datasets. Because there is no ground truth abundance and endmember data available on these three datasets, we only give qualitative evaluation to the abundance estimation by displaying the abundance maps generated by different methods. For the endmember evaluation on Urban and Jasper datasets, we generated the reference ground truth in the same way as in [40]. Ten spectra of each endmember were selected on the image and the mean value was then calculated as the ground truth endmember signature. For the Cuprite dataset, the ground truth endmembers were selected from the USGS library.

1) Urban HYDICE Dataset: The first dataset is the widely used urban image captured by the Urban Hyperspectral Digital Imagery Collection Experiment (HYDICE) sensor [40]. Each band is in the size of $307 \times 307$ pixels. The full image consists of 210 spectral channels with spectral resolution of $10 \mathrm{~nm}$ acquired in the range of $400 \mathrm{~nm}$ and $2500 \mathrm{~nm}$. After low SNR bands were removed (channels $1-4,76,87,101-111,136-153$, and 198-210), 162 bands were used 
TABLE VI

SADS OF DIFFERENT METHODS ON URBAN DATASET

\begin{tabular}{l|c|c|c}
\hline \hline Known Endmembers & 1 & 2 & 3 \\
\hline \hline NMF & $0.202 \pm 0.022$ & $0.202 \pm 0.022$ & $0.202 \pm 0.022$ \\
NMF-PPK (Unknown) & $0.191 \pm 0.019$ & $0.184 \pm 0.018$ & $0.181 \pm 0.019$ \\
NMF-PPK (All) & $0.188 \pm 0.018$ & $0.181 \pm 0.017$ & $0.179 \pm 0.018$ \\
\hline$L_{1 / 2}$-NMF & $0.166 \pm 0.014$ & $0.166 \pm 0.014$ & $0.166 \pm 0.014$ \\
$L_{1 / 2}$-PPK (Unknown) & $0.156 \pm 0.020$ & $0.151 \pm 0.024$ & $0.148 \pm 0.026$ \\
$L_{1 / 2}$-PPK (All) & $0.154 \pm 0.019$ & $0.147 \pm 0.022$ & $0.141 \pm 0.022$ \\
\hline MRS & $0.163 \pm 0.012$ & $0.163 \pm 0.012$ & $0.163 \pm 0.012$ \\
MRS-PPK (Unknown) & $0.156 \pm 0.019$ & $0.150 \pm 0.018$ & $0.147 \pm 0.019$ \\
MRS-PPK (All) & $0.155 \pm 0.018$ & $0.149 \pm 0.017$ & $0.147 \pm 0.018$ \\
\hline UPK-NMF (Unknown) & $0.225 \pm 0.031$ & $0.255 \pm 0.026$ & $0.295 \pm 0.035$ \\
UPK-NMF (All) & $0.169 \pm 0.025$ & $0.128 \pm 0.015$ & $0.074 \pm 0.010$ \\
\hline \hline
\end{tabular}

in the experiments, i.e., $H=162$.

In the experiment, we considered 4 types of endmembers: road, grass, asphalt, and tree [14]. We changed the number of known endmembers and identified the influence of the amount of prior knowledge. We ran this experiments 10 times with enumeration of the known endmembers and random initialization of the matrices. The mean and the standard deviation values of SAD of unknown endmembers with different extents of prior knowledge are displayed in Table VI. The results show that the proposed methods have led to significant improvement on the accuracy of endmember estimation, in both unknown and all endmembers, over the corresponding baseline methods. More prior knowledge can help in getting better endmember estimations in terms of smaller mean SAD values. UPK-NMF, on the contrary, performs significantly worse than other methods on unknown endmember estimations, its errors increasing when more endmembers are known. This is due to its limitations in dealing with discrepancy between prior knowledge and data. When such discrepancy is large, more errors are propagated into unknown endmembers. Its SADs of all endmember estimations improve when more endmembers are known. This is because in the current evaluation scheme, its error on known endmembers is zero. It should 


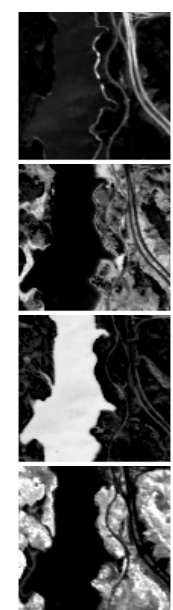

(a) $L_{1 / 2}-\mathrm{NMF}$

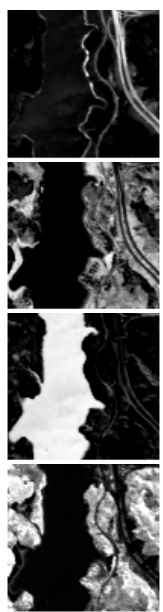

(b) $L_{1 / 2}-\mathrm{PPK}$

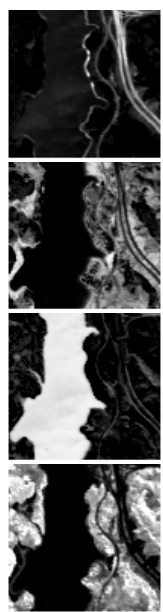

(c) MRS

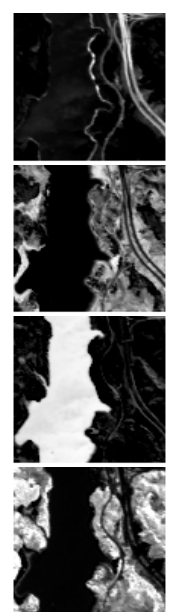

(d) MRS-PPK

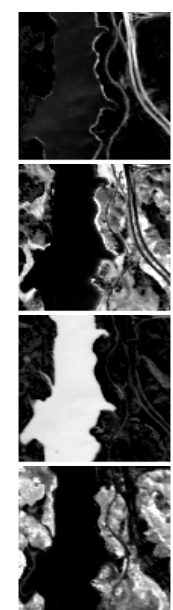

(e) UPK-NMF

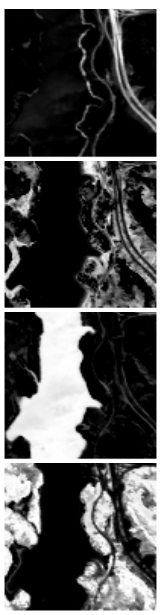

(f) CLSUNSAL

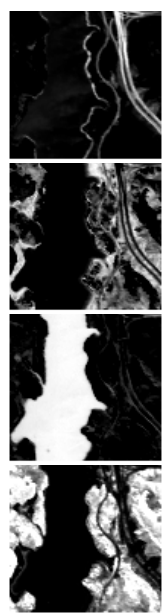

(g) SUNSPI

Fig. 7. Results of abundance estimation on the Jasper Ridge dataset. From top to bottom, rows show the abundance of Road, Soil, Water, and Tree, respectively.

be noted that the SADs of CLSUNSAL and SUNSPI are zero because they have included the ground truth signature in their libraries.

Fig. 6 shows the abundance maps of estimated endmembers, when road and grass are known. Each row represents the abundance of an endmember. From top to bottom, the figure shows the abundance of road, grass, asphalt, and tree. Each column stands for different methods. From left to right, they are the results from $L_{1 / 2}-\mathrm{NMF}, L_{1 / 2}-\mathrm{PPK}, \mathrm{MRS}, \mathrm{MRS}-\mathrm{PPK}$, UPKNMF, CLSUNSAL, and SUNSPI. We have removed the results of VCA because their SADs are significantly worse than the other approaches. From these abundance maps, it can be seen that all NMF based methods have produced quite similar results, except that UPK-NMF gives higher estimation on the grass abundance. Comparing with NMF based methods, the results from CLSUNSAL generated quite good estimates on grass and tree classes, but underestimates the road class. SUNSPI has significantly underestimates the abundance of road and asphalt.

2) Jasper Ridge Dataset: Now we turn our attention to the second real-world dataset: Jasper Ridge. It contains 224 bands covering the wavelengths from $380 \mathrm{~nm}$ to $2500 \mathrm{~nm}$, with a $10 \mathrm{~nm}$ spectral resolution. The size of a single band image is $512 \times 614$. In the experiments, we cut the original image to $100 \times 100$ subimage from pixel $(105,269)$ as in [41]. We have removed the low SNR and water-vapor absorption bands (1-3, 108-112, 154-166 and 220-224), which yielded 
TABLE VII

SADS OF DIFFERENT METHODS ON JASPER RIDGE DATASET

\begin{tabular}{l|c|c|c}
\hline \hline Known Endmembers & 1 & 2 & 3 \\
\hline \hline NMF & $0.108 \pm 0.003$ & $0.108 \pm 0.003$ & $0.108 \pm 0.003$ \\
NMF-PPK (Unknown) & $0.101 \pm 0.004$ & $0.095 \pm 0.004$ & $0.092 \pm 0.004$ \\
NMF-PPK (All) & $0.098 \pm 0.004$ & $0.090 \pm 0.004$ & $0.081 \pm 0.004$ \\
\hline$L_{1 / 2}$-NMF & $0.066 \pm 0.004$ & $0.066 \pm 0.004$ & $0.066 \pm 0.004$ \\
$L_{1 / 2}$-PPK (Unknown) & $0.062 \pm 0.004$ & $0.058 \pm 0.003$ & $0.054 \pm 0.004$ \\
$L_{1 / 2}$-PPK (All) & $0.060 \pm 0.003$ & $0.053 \pm 0.003$ & $0.049 \pm 0.003$ \\
\hline MRS & $0.065 \pm 0.003$ & $0.065 \pm 0.003$ & $0.065 \pm 0.003$ \\
MRS-PPK (Unknown) & $0.061 \pm 0.004$ & $0.057 \pm 0.004$ & $0.053 \pm 0.004$ \\
MRS-PPK (All) & $0.059 \pm 0.004$ & $0.052 \pm 0.004$ & $0.048 \pm 0.004$ \\
\hline UPK-NMF (Unknown) & $0.103 \pm 0.005$ & $0.102 \pm 0.008$ & $0.107 \pm 0.015$ \\
UPK-NMF (All) & $0.098 \pm 0.004$ & $0.051 \pm 0.004$ & $0.027 \pm 0.004$ \\
\hline \hline
\end{tabular}

198 bands out of the original 224. 4 types of endmembers were modelled in the experiments, i.e., road, soil, water and tree.

In the experiment, we also enumerated the combination of known endmembers and ran the code of each method 10 times. Similar to the results on the Urban HYDICE data, the results in Table VII also confirm that prior knowledge can help in getting better endmember estimations. The proposed methods marginally outperformed their counterparts NMF, $L_{1 / 2}$-NMF, and MRSNMF in SAD, and showed significant advantages over UPK-NMF in unknown endmember estimation. Fig. 7 shows the abundance maps of four endmembers when road and soil are set as the prior knowledge. Each row represents the abundance of an endmember. From top to bottom, they are the abundances of road, soil, water and tree. Each column stands for different methods. From left to right, they are $L_{1 / 2}-\mathrm{NMF}, L_{1 / 2}$-PPK, MRS, MRS-PPK, UPK-NMF, CLSUNSAL, and SUNSPI. All methods have shown quite similar estimation results.

3) Cuprite Dataset: Finally, we show the results on Cuprite dataset which was captured by AVIRIS sensor in Souther Nevada. It is a regional scene which contains an abundant supply of minerals. The original data is with size $250 \times 190$. For our experiments, we have removed low 
TABLE VIII

SADS OF DIFFERENT METHODS ON CUPRITE DATA SET

\begin{tabular}{l|c|c|c}
\hline \hline Known Endmember & 1 & 2 & 3 \\
\hline \hline NMF & $0.198 \pm 0.021$ & $0.198 \pm 0.021$ & $0.198 \pm 0.021$ \\
NMF-PPK (Unknown) & $0.193 \pm 0.023$ & $0.189 \pm 0.021$ & $0.188 \pm 0.020$ \\
NMF-PPK (All) & $0.190 \pm 0.020$ & $0.181 \pm 0.021$ & $0.178 \pm 0.020$ \\
\hline$L_{1 / 2}$-NMF & $0.165 \pm 0.012$ & $0.165 \pm 0.012$ & $0.165 \pm 0.012$ \\
$L_{1 / 2}$-PPK (Unknown) & $0.161 \pm 0.009$ & $0.160 \pm 0.012$ & $0.159 \pm 0.008$ \\
$L_{1 / 2}$-PPK (All) & $0.158 \pm 0.010$ & $0.157 \pm 0.011$ & $0.153 \pm 0.009$ \\
\hline MRS & $0.163 \pm 0.014$ & $0.163 \pm 0.014$ & $0.163 \pm 0.014$ \\
MRS-PPK (Unknown) & $0.159 \pm 0.015$ & $0.158 \pm 0.016$ & $0.158 \pm 0.013$ \\
MRS-PPK (All) & $0.155 \pm 0.014$ & $0.153 \pm 0.015$ & $0.151 \pm 0.012$ \\
\hline UPK-NMF (Unknown) & $0.211 \pm 0.024$ & $0.216 \pm 0.017$ & $0.220 \pm 0.025$ \\
UPK-NMF (All) & $0.190 \pm 0.022$ & $0.173 \pm 0.016$ & $0.154 \pm 0.023$ \\
\hline \hline
\end{tabular}

SNR and water-vapor absorption bands (1-2, 104-113, 148-167 and 221-224), which yields 188 bands out of the original 224 bands. We compared the SAD estimation of 10 types of minerals, i.e., Alunite, Andradite, Buddingtonite, Dumortierite, Kaolinite, Montmorillonite, Muscovite, Nontronite, Pyrope, and Sphene.

Table VIII shows the results from different methods. Note that in this experiment, we randomly selected known endmembers rather than trying all combinations because the number of endmembers is much higher than the previous two datasets. It can be seen that the proposed method outperformed the NMF, $L_{1 / 2}$-NMF and MRS-NMF methods on all endmember estimations. The SADs on unknown endmembers are marginally better than the baselines. This is due to the high discrepancy between known endmembers and the data. Such discrepancy can also be proven by the results of UPK-NMF which has generated higher errors than other methods on the unknown endmember estimation. 


\section{Conclusion}

In this paper, we have described a NMF based unmixing method which deals with partially known endmembers. This method tries to minimize the differences between the estimated endmembers and the known endmembers. It has the capability of dealing with the discrepancies between the estimated endmembers and the prior knowledge. Moreover, the proposed method is general in nature and can be extended to other NMF based methods with various constraints in the estimation models. A series of experiments on both synthetic and real data show that the proposed method has not only achieved better performance than several baseline methods that do not use prior knowledge, but has also outperformed an alternative unmixing method that also uses partial knowledge. The proposed method also performs better than the sparse regression approach, which relies on an over-complete dictionary of endmembers, when key endmembers are missing in the dictionary. In the future, we will explore fusion of prior knowledge with other hyperspectral unmixing methods.

\section{ACKNOWLEDGEMENT}

This work was partly supported by the Australian Research Council DECRA Projects funding scheme under Project DE120102948, the National Natural Science Foundation of China under Grant 61370123 and Grant 61571393, and Beijing Natural Science Foundation Project 4162037.

\section{REFERENCES}

[1] A. F. Goetz, G. Vane, J. E. Solomon, and B. N. Rock, "Imaging spectrometry for earth remote sensing," Science, vol. 228, no. 4704, pp. 1147-1153, 1985.

[2] M. Fauvel, J. A. Benediktsson, J. Chanussot, and J. R. Sveinsson, "Spectral and spatial classification of hyperspectral data using svms and morphological profiles," IEEE Transactions on Geoscience and Remote Sensing, vol. 46, no. 11, pp. 3804-3814, 2008.

[3] C. C. Lelong, P. C. Pinet, and H. Poilvé, "Hyperspectral imaging and stress mapping in agriculture: a case study on wheat in beauce (france)," Remote Sensing of Environment, vol. 66, no. 2, pp. 179-191, 1998.

[4] R. Resmini, M. Kappus, W. Aldrich, J. Harsanyi, and M. Anderson, "Mineral mapping with hyperspectral digital imagery collection experiment (HYDICE) sensor data at Cuprite, Nevada, USA," International Journal of Remote Sensing, vol. 18, no. 7, pp. 1553-1570, 1997.

[5] N. Keshava and J. F. Mustard, "Spectral unmixing," IEEE Signal Processing Magazine, vol. 19, no. 1, pp. 44-57, 2002.

[6] J. M. Bioucas-Dias, A. Plaza, N. Dobigeon, M. Parente, Q. Du, P. Gader, and J. Chanussot, "Hyperspectral unmixing overview: Geometrical, statistical, and sparse regression-based approaches," IEEE Journal of Selected Topics in Applied Earth Observations and Remote Sensing, vol. 5, no. 2, pp. 354-379, 2012. 
[7] N. Keshava, "A survey of spectral unmixing algorithms," Lincoln Laboratory Journal, vol. 14, no. 1, pp. 55-78, 2003.

[8] M. E. Winter, "N-FINDR: an algorithm for fast autonomous spectral end-member determination in hyperspectral data," in SPIE International Symposium on Optical Science, Engineering, and Instrumentation, 1999, pp. 266-275.

[9] J. W. Boardman, "Automating spectral unmixing of aviris data using convex geometry concepts," in Summaries 4th Annual JPL Airborne Geoscience Workshop, vol. 1, 1993, pp. 11-14.

[10] J. M. Nascimento and J. M. Dias, "Vertex component analysis: A fast algorithm to unmix hyperspectral data," IEEE Transactions on Geoscience and Remote Sensing, vol. 43, no. 4, pp. 898-910, 2005.

[11] J. M. Bioucas-Dias, “A variable splitting augmented lagrangian approach to linear spectral unmixing," in First Workshop on Hyperspectral Image and Signal Processing: Evolution in Remote Sensing., 2009, pp. 1-4.

[12] V. P. Pauca, J. Piper, and R. J. Plemmons, "Nonnegative matrix factorization for spectral data analysis," Linear Algebra and its Applications, vol. 416, no. 1, pp. 29-47, 2006.

[13] L. Miao and H. Qi, "Endmember extraction from highly mixed data using minimum volume constrained nonnegative matrix factorization,” IEEE Transactions on Geoscience and Remote Sensing, vol. 45, no. 3, pp. 765-777, 2007.

[14] S. Jia and Y. Qian, "Constrained nonnegative matrix factorization for hyperspectral unmixing," IEEE Transactions on Geoscience and Remote Sensing, vol. 47, no. 1, pp. 161-173, 2009.

[15] Y. Qian, S. Jia, J. Zhou, and A. Robles-Kelly, "Hyperspectral unmixing via $L_{1 / 2}$ sparsity-constrained nonnegative matrix factorization," IEEE Transactions on Geoscience and Remote Sensing, vol. 49, no. 11, pp. 4282-4297, 2011.

[16] X. Lu, H. Wu, Y. Yuan, P. Yan, and X. Li, “Manifold regularized sparse nmf for hyperspectral unmixing," IEEE Transactions on Geoscience and Remote Sensing, vol. 51, no. 5, pp. 2815-2826, 2013.

[17] J. Liu, J. Zhang, Y. Gao, C. Zhang, and Z. Li, "Enhancing spectral unmixing by local neighborhood weights," IEEE Journal of Selected Topics in Applied Earth Observations and Remote Sensing, vol. 5, no. 5, pp. 1545-1552, 2012.

[18] N. Wang, B. Du, and L. Zhang, "An endmember dissimilarity constrained non-negative matrix factorization method for hyperspectral unmixing," IEEE Journal of Selected Topics in Applied Earth Observations and Remote Sensing, vol. 6, no. 2, pp. 554-569, 2013.

[19] R. Clark, G. Swayze, R. Wise, E. Livo, T. Hoefen, R. Kokaly, and S. Sutley, "USGS digital spectral library splib06a: U.S. Geological Survey, Digital Data Series 231.” USGS, 2007.

[20] D. Roberts, M. Gardner, R. Church, S. Ustin, G. Scheer, and R. Green, "Mapping chaparral in the santa monica mountains using multiple endmember spectral mixture models," Remote Sensing of Environment, vol. 65, no. 3, p. $267279,1998$.

[21] M.-D. Iordache, J. Bioucas-Dias, and A. Plaza, "Sparse unmixing of hyperspectral data," IEEE Transactions on Geoscience and Remote Sensing, vol. 49, no. 6, pp. 2014-2039, June 2011.

[22] J. M. Bioucas-Dias and M. A. Figueiredo, "Alternating direction algorithms for constrained sparse regression: Application to hyperspectral unmixing," in 2nd Workshop on Hyperspectral Image and Signal Processing: Evolution in Remote Sensing, 2010, pp. 1-4.

[23] M. Iordache, J. Dias, and A. Plaza, "Collaborative sparse regression for hyperspectral unmixing," IEEE Transactions on Geoscience and Remote Sensing, vol. 52, no. 1, pp. 341-354, 2014.

[24] _ _ "Total variation spatial regularization for sparse hyperspectral unmixing," IEEE Transactions on Geoscience and Remote Sensing, vol. 50, no. 11, pp. 4484-4502, 2012.

[25] N. Akhtar, F. Sahfait, and A. Mian, "Repeated constrained sparse coding with partial dictionaries for hyperspectral unmixing," in IEEE Winter Conference on Applications of Computer Vision, 2014, pp. 953-960.

[26] X. Fu, W.-K. Ma, J. M. Bioucas-Dias, and T.-H. Chan, "A robust subspace method for semiblind dictionary-aided 
hyperspectral unmixing," in Workshop on Hyperspectral Image and Signal Processing: Evolution in Remote Sensing, 2014.

[27] M.-D. Iordache, J. Bioucas-Dias, and A. Plaza, "MUSIC-CSR: Hyperspectral unmixing via multiple signal classification and collaborative sparse regression," IEEE Transactions on Geoscience and Remote Sensing, vol. 52, no. 2, pp. 4364-4382, 2014.

[28] W. Tang, Z. Shi, and Z. An, "Nonnegative matrix factorization for hyperspectral unmixing using prior knowledge of spectral signatures," Optical Engineering, vol. 51, no. 8, pp. 087001-1, 2012.

[29] W. Tang, Z. Shi, Y. Wu, and C. Zhang, "Sparse unmixing of hyperspectral data using spectral a priori information," IEEE Transactions on Geoscience and Remote Sensing, vol. 53, no. 2, pp. 770-783, 2015.

[30] J. Sigurdsson, M. O. Ulfarsson, and J. R. Sveinsson, "Endmember constrained semi-supervised hyperspectral unmixing," in Workshop on Hyperspectral Image and Signal Processing: Evolution in Remote Sensing, 2014.

[31] D. D. Lee and H. S. Seung, "Learning the parts of objects by non-negative matrix factorization," Nature, vol. 401 , no. 6755, pp. 788-791, 1999.

[32] — - "Algorithms for non-negative matrix factorization," in Advances in Neural Information Processing Systems, 2000, pp. 556-562.

[33] A. P. Dempster, N. M. Laird, D. B. Rubin et al., "Maximum likelihood from incomplete data via the em algorithm," Journal of the Royal statistical Society, vol. 39, no. 1, pp. 1-38, 1977.

[34] L. Saul and F. Pereira, "Aggregate and mixed-order markov models for statistical language processing," in Proceedings of the Second Conference on Empirical Methods in Natural Language Processing, 1997, pp. 81-89.

[35] C.-J. Lin, "On the convergence of multiplicative update algorithms for nonnegative matrix factorization," IEEE Transactions on Neural Networks, vol. 18, no. 6, pp. 1589-1596, 2007.

[36] E. F. Gonzalez and Y. Zhang, "Accelerating the Lee-Seung algorithm for non-negative matrix factorization,” Dept. Comput. \& Appl. Math., Rice Univ., Houston, TX, Tech. Rep. TR-05-02, 2005.

[37] D. C. Heinz et al., "Fully constrained least squares linear spectral mixture analysis method for material quantification in hyperspectral imagery," IEEE Transactions on Geoscience and Remote Sensing, vol. 39, no. 3, pp. 529-545, 2001.

[38] T. H. Cormen, C. E. Leiserson, R. L. Rivest, C. Stein et al., Introduction to algorithms. MIT press Cambridge, 2001, vol. 2.

[39] P. O. Hoyer, "Non-negative matrix factorization with sparseness constraints," The Journal of Machine Learning Research, vol. 5, pp. 1457-1469, 2004.

[40] S. Jia and Y. Qian, "Spectral and spatial complexity-based hyperspectral unmixing," IEEE Transactions on Geoscience and Remote Sensing, vol. 45, no. 12, pp. 3867-3879, 2007.

[41] F. Zhu, Y. Wang, S. Xiang, B. Fan, and C. Pan, "Structured sparse method for hyperspectral unmixing," ISPRS Journal of Photogrammetry and Remote Sensing, vol. 88, pp. 101 - 118, 2014. 